\title{
Aspectos regionales de la movilidad social y la igualdad de oportunidades en Colombia*
}

Recibido: 21 de enero de 2014 - Aceptado: 11 de febrero de 2015

Doi: dx.doi.org/10.12804/rev.econ.rosario.17.02.2014.03

Luís Armando Galvis-Aponte ${ }^{\dagger}$

Adolfo Meisel-Roca $\ddagger$

Resumen

El objetivo de la presente investigación es estudiar la movilidad social en Colombia en el ámbito regional. Colombia tiene uno de los más altos índices de concentración del ingreso en el mundo y amplias disparidades económicas a nivel regional, que han sido persistentes en el tiempo. En este trabajo se estudia uno de los factores determinantes de dicha persistencia: baja movilidad social de las generaciones. Los resultados confirman los bajos niveles de movilidad intergeneracional en la educación, aunque se reportan mejorías. Además, se encuentra una fuerte asociación entre el ingreso de una región y el grado de movilidad social. También, hay una correlación negativa y significativa entre el grado de desigualdad en los ingresos de una región y el grado de movilidad social. Esta investigación representa un primer esfuerzo

* Los autores agradecen los comentarios de Jaime Bonet, Javier Pérez, Karelys Guzmán, Leonardo Bonilla y Mónica Gómez. Se agradece la invaluable asistencia de Lina Moyano, estudiante en práctica del CEER, así como a Bladimir Carrillo. Se agradece también a Jérémie Gignoux por compartir sus programas para los cálculos de los índices de desigualdad de oportunidades. Para comentarios, favor dirigirse al correo electrónico: lgalviap @ banrep.gov.co

+ Economista del Centro de Estudios Económicos Regionales - CEER-, Banco de la República, sucursal Cartagena.

$\ddagger$ Codirector del Banco de la República.

Para citar este artículo: Galvis-Aponte, L. A., \& Meisel-Roca, A. (2014). Aspectos regionales de la movilidad social y la igualdad de oportunidades en Colombia. Revista de Economía del Rosario, 17(2), 257-297. doi: dx.doi.org/10.12804/rev.econ.rosario.17.02.2014.03 
por relacionar las condiciones de movilidad social con las de desigualdad, avanzando en el estudio de la transmisión intergeneracional de las desigualdades en Colombia.

Clasificación JEL: D31, E24, I21, J62

Palabras clave: movilidad social, desigualdad de oportunidades.

\title{
Regional Aspects of Social Mobility and Equality of Opportunities in Colombia
}

\begin{abstract}
This paper studies social mobility in Colombia at a regional level, for 2003 and 2010. Colombia has one of the highest levels of income concentration in the world. It also has significant regional economic disparities. Both the high level of regional economic disparities and the income concentration have been highly persistent. In this paper we study one of the determinants of that persistence: low levels of social mobility across the generations. The results of this paper confirm those low levels of intergenerational mobility in education. However, comparing the years 2003 with 2010 we notice that there have been improvements in this regard. The results also reveal a strong association between the level of income of a region and the degree of social mobility it presents. There is also a negative and significant correlation between the degree of income inequality of a region and the extent of social mobility. This research represents a first attempt to study the relation between conditions of social mobility with inequalities, to advance in the study of the intergenerational transmission of inequality in Colombia.
\end{abstract}

JEL Classification: D31, E24, I21, J62

Keywords: social mobility, inequality of opportunities.

\section{Aspetos regionais da mobilidade social e a igualdade de oportunidades na Colômbia}

\author{
Resumo
}

O objetivo da presente pesquisa é estudar a mobilidade social na Colômbia no nível regional. A Colômbia tem um dos maiores índices de concentração do ingresso no mundo, e amplas disparidades económicas no nível regional, que têm sido persistentes através do tempo. Neste trabalho estuda-se um dos fatores determinantes de dita persistência: baixa mobilidade social através das gerações. Os resultados confirmam os baixos níveis de mobilidade intergeracional na educação, ainda que reportam-se melhoras. Além disso, encontra-se uma forte associação entre o ingresso de uma região e o grau de mobilidade social. Também há uma correlação negativa e significativa entre o grau de desigualdade nos ingressos de uma região e o grau de mobilidade social. Esta pesquisa representa um primeiro esforço por relacionar as condições de mobilidade social com as de desigualdade, avançando no estudo da transmissão intergeracional das desigualdades na Colômbia.

Classificação JEL: D31, E24, I21, J62

Palavras-chave: mobilidade social, desigualdade de oportunidades. 


\section{Introducción}

La persistencia de las condiciones de pobreza y desigualdad ha sido una de las características de las regiones y municipios de Colombia. Durante las últimas tres décadas, los niveles de pobreza permanecieron relativamente inmutables, ya que existe una estrecha relación entre el porcentaje de personas pobres que se reportó en los censos de 1973 y 2005 (Galvis \& Meisel, 2010). Las condiciones de desigualdad no solo se reflejan en las altas cifras del coeficiente de Gini, sino que son evidentes además en otras medidas que también dan cuenta de las disparidades en el acceso a oportunidades. Por ejemplo, Barros et al. (2009) reportan que en Colombia un estudiante que proviene de una familia aventajada ${ }^{1}$ tiene una probabilidad del $95 \%$ de terminar sexto grado a tiempo, mientras que dicha probabilidad para un niño proveniente de una familia con desventajas en su nivel socioeconómico llega al $15 \%$. En este caso, el niño aventajado tiene una probabilidad seis veces mayor de completar sexto grado, que la del niño con desventajas. En contraste, para Chile, la razón entre dichas probabilidades es de dos a uno en favor del niño aventajado.

Esas desigualdades en el acceso de oportunidades no son capturadas por los índices de desigualdad global, ni mucho menos por los índices de pobreza. Más bien, la pobreza exacerba las condiciones de desigualdad, en tanto que las características socioeconómicas de los hogares pueden ser un determinante fundamental del probable éxito de las generaciones que apenas están empezando su trasegar por la vida. Los estudios de movilidad social en Colombia han coincidido en mostrar que dicho fenómeno tiene una muy baja ocurrencia en el país. Sin embargo, surge la pregunta de cómo esa baja movilidad está relacionada con la igualdad de oportunidades.

En general, la ausencia de movilidad social puede ser el resultado de situaciones no equitativas o que no reflejan el esfuerzo de los individuos. Visto de otra manera, "la movilidad social no siempre está asociada de manera unívoca con la igualdad de oportunidades y mucho menos con la meritocracia" (Angulo et al., 2012, p. 3).

El presente documento tiene como objetivo estudiar la movilidad social vista desde una perspectiva regional. Se llevan a cabo ejercicios que consisten en

1 La condición de aventajado o con desventajas viene definida subjetivamente, considerándose aventajado un niño que tiene un solo hermano y que proviene de un hogar con la presencia del padre y la madre, ambos con educación secundaria completa y que devengan un ingreso per cápita de US $\$ 25$ diarios de 2005, ajustados por paridad. Los niños con desventajas se considera que son los que tienen cuatro hermanos, que provienen de hogares del área rural en donde solo está presente uno de los padres, que es analfabeta y que recibe un ingreso de US\$1 por día. 
calcular (a) índices de movilidad intergeneracional en educación, promediados para el grupo de edad entre 25 y 65 años; (b) movilidad por cuantiles, según los años de educación, lo cual permite indagar por las posibilidades de movilidad intergeneracional, de acuerdo con la posición en la distribución de los años de educación; (c) movilidad en el Índice de Condiciones Materiales (ICM), calculado a partir de la metodología de los conjuntos borrosos; (d) relación entre la movilidad y la desigualdad de oportunidades, siguiendo la propuesta de Ferreira y Gignoux (2011). Esta última sección representa un primer esfuerzo por relacionar las condiciones de movilidad social con las de desigualdad, más allá del uso del tradicional coeficiente de Gini. De esta manera, se avanza entonces en el estudio de la transmisión intergeneracional de las desigualdades en Colombia.

En el primer capítulo se introduce el tema. En el segundo se presenta una revisión de la literatura relevante. En el tercero se describe sucintamente la metodología que se emplea. En el cuarto capítulo se presentan los resultados en torno a la movilidad social y la igualdad de oportunidades. En el quinto se concluye.

\section{Revisión de literatura}

En relación con el estudio de la movilidad social, el trabajo de Gaviria (2002) es uno de los primeros en Colombia en incursionar en esta materia. Además de construir indicadores de movilidad social, ese estudio indaga por el papel de la educación como herramienta para salir de la pobreza. El autor también analiza estos tópicos desde una perspectiva regional. La metodología empleada para construir índices de movilidad social es una regresión simple, en donde la variable dependiente es la educación de los hijos y la variable independiente, los años de educación del padre. Adicionalmente, el autor construye matrices de transición y compara los resultados con otros países (México, Brasil, Perú y Estados Unidos). Los datos para Colombia provienen de la Encuesta Nacional de Calidad de Vida (ECV) de 1997. Los resultados indican que, frente a los demás países analizados, la movilidad en Colombia es baja. Por último, los hallazgos sugieren disparidades regionales en Colombia en la movilidad social, siendo esta última mayor en Bogotá. Estos hallazgos, sin embargo, deben ser interpretados con cautela. Al utilizar índices de movilidad en educación, provenientes de regresiones simples, el autor no controla por la dispersión de la educación. En tal sentido, es imposible determinar si las diferencias en movilidad se deben a la correlación entre los años de educación o a la dispersión de los años de educación.

Analizando un periodo más amplio, Nina et al. (2003) buscan probar la hipótesis de que la movilidad social está vinculada con el nivel educativo. El análisis 
se lleva a cabo para la ciudad de Bogotá. La metodología empleada consiste en estimar matrices de transición y con ellas calcular el índice propuesto por Shorrocks (1978). La información empleada proviene de la Encuesta Nacional de Hogares (1978-1998) y de la ECV (1997). La principal conclusión a la que se llega es que existe una mayor movilidad en la medida en que se analizan grupos de la población que comenzaron con un nivel educativo más bajo.

A diferencia de los estudios previos, Cartagena (2003) calculó un nuevo índice de movilidad que se basa en la probabilidad neta de que el nivel de escolaridad de los hijos supere al de sus padres. Los resultados son consistentes en mostrar que el grado de movilidad social es más alto entre segmentos de la población que empezaron, en promedio, con un nivel más bajo de educación. El estudio utiliza datos de la Encuesta de Calidad de Vida (ECV) de 1997 para investigar la movilidad social. La autora emplea matrices de transición para analizar la movilidad educativa y ocupacional entre padres e hijos y entre parejas. $\mathrm{Al}$ igual que otros estudios hechos en el país, se concluye que la movilidad es baja y que coincide con una alta desigualdad de ingresos.

Adicionalmente, la autora estima los retornos a la educación en una ecuación tipo Mincer por niveles de escolaridad. Con esto último, se intenta mostrar que las diferencias en la movilidad social están relacionadas con diferencias en los retornos a la educación. Los resultados del estudio tienden a confirmar este planteamiento. Sin embargo, al estimar los retornos a la educación, se ignora un problema conocido: la endogeneidad de la educación provocada por la existencia de variables no observadas que se relacionan tanto con el salario como con los años de educación de los individuos.

Un estudio que analiza a fondo las diferencias regionales en la movilidad intergeneracional en educación es el de Bonilla (2010). A diferencia de estudios previos, este autor calcula varias clases de indicadores de movilidad para diferentes ciudades y regiones. En concreto, el autor emplea siete índices de movilidad, unos basados en matrices de transición y otros apoyados en regresiones lineales por Mínimos Cuadrados Ordinarios (MCO). Los datos utilizados provienen de la Gran Encuesta Integrada de Hogares (GEIH) y de la ECV. Los principales hallazgos son que existe menos movilidad en ciudades tales como Bogotá, Cartagena y Cali, mientras que ocurre lo contrario en ciudades como Neiva y Valledupar. Del mismo modo, se encuentra que existe mayor movilidad en la zona rural en relación con la zona urbana. También se muestra que las mujeres tienen mayor movilidad que los hombres. El autor, sin embargo, señala que existe alta sensibilidad al tipo de índice usado. Mientras que algunos indicadores sugieren mayor movilidad a mayor educación, otros índices sugieren justo lo contrario. Como el autor lo indica, esta variación en los resultados sugiere que para adelantar estudios de movilidad es necesario construir varios indicadores. 
Utilizando los datos de la Encuesta Nacional de Hogares para Bogotá, Cali y el agregado de las trece principales ciudades, Viáfara et al. (2010) estudian la movilidad intergeneracional educativa teniendo en cuenta el origen étnicoracial. La metodología se basa en modelos de Markov y matrices de transición. Los principales hallazgos de los autores muestran que la población de origen afrocolombiano registra una movilidad más baja que sus pares no afrocolombianos. Estas disparidades tienden a profundizarse en Bogotá.

Londoño (2011) analiza los determinantes de la percepción de movilidad pasada y las expectativas de movilidad futura. Adicionalmente, el estudio también investiga el efecto de las percepciones de movilidad y justicia social sobre el bienestar subjetivo. La fuente de datos empleada proviene de la Encuesta Social y Política (ESP) de la Universidad de los Andes e Invamer Gallup de 2007. Los resultados indican que el ingreso ejerce un efecto positivo sobre el nivel de bienestar individual. También se encuentra que la expectativa de movilidad futura no ejerce influencia sobre el bienestar individual. Este hallazgo, sin embargo, no puede ser interpretado como un efecto causal, debido a que pueden existir factores inobservables que afecten tanto la expectativa de movilidad futura como el bienestar individual.

Ferreira y Meléndez (2012) hacen un análisis de la desigualdad en Colombia para el periodo 1997-2010. Con este propósito, los autores utilizan las ECV de 1997, 2003, 2008 y 2010. Los resultados indican que, en relación con otros países de América Latina, la desigualdad en el país es alta. Los autores muestran que esta situación está determinada en parte por las circunstancias al nacer de los individuos. Específicamente, se muestra que los individuos que son hijos de padres con bajo nivel educativo se encuentran con mayores desventajas, con respecto a aquellos que poseen padres con mayor capital humano. Esto sugiere claramente que la alta desigualdad en el país está asociada con una baja movilidad social intergeneracional. También se evidencia que los individuos oriundos de regiones como el Caribe se encuentran en desventaja en términos de igualdad de oportunidades. Esto quiere decir, como ya se ha anotado en los estudios mencionados, que la movilidad social intergeneracional no se distribuye homogéneamente entre las regiones de Colombia.

Un estudio en donde se reconoce la importancia del papel de la primera infancia en la movilidad social es el desarrollado por Bernal y Camacho (2012). Las autoras señalan que la experiencia internacional documenta los altos beneficios, en términos sociales, de intervenir en la formación de capital humano en las primeras etapas del ciclo vital. Uno de los hallazgos más importantes del diagnóstico realizado por las autoras para Colombia es la evidencia sobre las tasas de embarazo juvenil, significativamente más altas entre la población más pobre. Estas disparidades son importantes, en vista de que la fecundidad 
juvenil es un determinante esencial de la formación del capital humano en las primeras etapas de la vida. Además, trae como consecuencia baja movilidad social y mayor desigualdad. En este contexto, las autoras recomiendan promover los programas de asistencia a la primera infancia con el fin de mitigar las desventajas iniciales de la población más pobre y así romper las trampas de pobreza.

Empleando la Encuesta de Calidad de Vida y Movilidad Social (ECVMS) de 2010, Angulo et al. (2012) llevan a cabo un análisis de la movilidad socioeconómica en Colombia, utilizando nuevos módulos que hacen preguntas retrospectivas sobre las características socioeconómicas de los padres. Al igual que en otros estudios para Colombia, el cálculo de los índices de movilidad se basa en regresiones lineales y matrices de transición. Los resultados confirman que la movilidad social en el país es baja cuando se compara con otros países de América Latina. Sin embargo, los autores señalan que la movilidad ha aumentado. Por último, los autores sugieren la existencia de una persistencia en la desigualdad que es concomitante con la baja movilidad en el país.

En el contexto Latinoamericano, se encuentran estudios de movilidad en educación y el logro laboral para Brasil, Colombia, México y Perú. En Behrman et al. (2001), los indicadores de movilidad de estos países son comparados con los correspondientes a los obtenidos para Estados Unidos. Los autores usan encuestas de hogares con información retrospectiva, dada la ausencia de encuestas longitudinales. La metodología empleada se basa en regresiones lineales simples. Los principales resultados muestran que (a) la movilidad es considerablemente más alta en Estados Unidos que en Latinoamérica; (b) existen disparidades entre este grupo de países; (c) Colombia y Brasil son los países que registran más baja movilidad social; (d) las áreas urbanas tienden a presentar mayor movilidad que las rurales; (e) por género, se evidencia que los hombres registran mayor movilidad que las mujeres; (f) la movilidad social ha venido aumentando con el paso del tiempo.

Dahan y Gaviria (1999) investigan la movilidad educativa para dieciséis países de América Latina y Estados Unidos. A diferencia de otros estudios para la región, estos autores proponen un nuevo índice de movilidad que se basa en las oportunidades sociales de los individuos jóvenes que aún residen con sus padres. En este estudio se encuentra que Estados Unidos y Costa Rica son los que registran mayor movilidad. Colombia y México tienden a ser los que presentan menor movilidad. Los autores reconocen las desventajas del índice propuesto, entre estas se mencionan la calidad de la educación, que no es tenida en cuenta en la nueva metodología. Sin embargo, concluyen que esta nueva aproximación puede brindar información relevante especialmente en países en desarrollo, donde los problemas de cobertura educativa persisten. 
Espinoza et al. (2009), utilizando datos para los jóvenes de Chile, investigan el vínculo entre educación, resultados en el mercado laboral y origen socioeconómico. Una ventaja del estudio es que también usa datos de fuentes primarias para la ciudad de Santiago. La metodología empleada se basa en matrices de transición. Lo que se encuentra es que existe un fuerte vínculo entre el nivel educativo de los padres e hijos, lo cual sugiere poca movilidad social. Lo mismo se tiende a encontrar cuando se analiza la movilidad en el nivel ocupacional.

Torche y Spilerman (2009) tratan el tema en México utilizando la Encuesta de Movilidad Social de 2006. Los autores estiman el efecto del estatus económico de los padres sobre el logro educacional, el consumo, tenencia de activos, propiedad y el valor de la vivienda muestran que los resultados de los jóvenes, en términos educativos y otros indicadores socioeconómicos, están estrechamente vinculados a la riqueza de los padres. Este vínculo es más fuerte para la población residente en las áreas rurales y la población de más bajos recursos.

Eide y Showalter (1999) parten de reconocer que existe heterogeneidad en las medidas de movilidad basadas en regresiones lineales, ante lo cual proponen utilizar regresiones por cuantiles para tener en cuenta la variación en estos índices. Como ilustración empírica, los autores utilizan datos longitudinales para el periodo 1968-1992 de Estados Unidos. Los resultados indican que la correlación entre ingresos intergeneracionales es mayor en el fondo de la distribución condicional de ingresos que en la cima de esta. Asimismo, se encuentra que si se controla por el nivel educativo, se reduce la correlación intergeneracional en ingresos. También se encuentra que el papel de la educación es más apreciable en la parte baja de la curva de distribución de ingresos.

En el presente documento se estudia la movilidad intergeneracional en educación y en condiciones materiales de vida. El estudio se desvía de la literatura existente en Colombia en varios aspectos. En primer lugar, se realiza un análisis por cuantiles para caracterizar las posibilidades de movilidad en diferentes niveles de logro educativo. También se hace distinción entre la movilidad ascendente y descendente. Por último, se enfatiza en la relación de la movilidad social con las condiciones de los individuos en términos de la igualdad de oportunidades.

\section{Metodología}

\subsection{Medición de movilidad}

El concepto de movilidad social tiene su origen en la sociología, en donde se inició al relacionar la situación de los hijos con la de sus padres. Se comparan situaciones de ahora con las que tenían los padres en el pasado y con edades 
equivalentes a las de los hijos. Cuando se observan mejorías en la situación de los hijos, existe movilidad social ascendente y viceversa. En el caso en que se mantengan condiciones similares, existe inmovilidad, o una situación de dependencia del logro de los hijos frente al de sus padres. En términos empíricos, se emplean variables como el nivel educativo, indicadores de condiciones socioeconómicas, de riqueza material, entre otros.

En términos metodológicos, el estudio de Prais (1955) es de los más citados, al ser uno de los primeros en utilizar matrices de transición para estudiar la movilidad social intergeneracional. En general, este método consiste en estimar la probabilidad de pasar de un estado a otro en el transcurso de un periodo determinado. En términos de movilidad social, esta estrategia puede emplearse, por ejemplo, para estimar la probabilidad de que una generación en un nivel educativo dado, en el tiempo $t$ pase a un nivel educativo más alto en el tiempo $t+1$. Formalmente, sea $X$ una matriz de dimensión $N \times 1$, que contiene la proporción de individuos en la clase $i$ en el tiempo $t$ y $P$ la matriz de transición $N \times$ $N$, entonces la distribución final de clase social $n$ periodos más tarde es igual a:

$$
X_{t+n}=P^{n} X_{t}
$$

La aplicación de la ecuación (1) supone que la matriz de transición permanece constante en el tiempo. Las clases pueden ser niveles educativos, quintiles de ingreso, estatus ocupacional, entre otros. Una propiedad de la matriz $P$ es que la suma de todos los elementos de cada fila es igual a 1, pues esta resulta de calcular la frecuencia relativa de los componentes que en el período $t$ se encontraban en la primera clase, de acuerdo con la posición que ocupan en el periodo $t+1$. El cálculo de $P$ también puede aproximarse a partir de la distribución de frecuencias de la población de interés. La estimación de matrices de transición provee información útil sobre el grado de movilidad social. No obstante, la comparación entre diferentes poblaciones se dificulta al no resumir el grado de movilidad en un índice.

\subsubsection{Método de regresión por $\mathrm{MCO}$}

Además de usar matrices de transición para medir la movilidad, también ha sido común estimar regresiones lineales simples. En este enfoque se busca medir la correlación entre el logro social de los hijos con el de los padres. Formalmente, la literatura en general ha estimado una ecuación de la forma:

$$
y_{t}=\alpha+\beta y_{t-1}+u_{t}
$$


Donde $y_{t}$ representa el logaritmo, ya sea de los años de educación, alguna medida de riqueza o los ingresos, del hijo $(t)$ y del padre $(t-1)$. El término $u$ es el error que se supone aleatoriamente distribuido. El parámetro $\beta$ mide el grado de movilidad, pero en orden inverso, es decir que cuanto mayor sea este término menor será la movilidad. Por lo anterior, se define alternativamente el índice gamma, que se calcula como $(1-\beta)$.

En situación de perfecta movilidad el parámetro $\beta$ es igual a 0 . Es decir, en condiciones de igualdad de oportunidades el éxito económico de la presente generación no debería ser determinado por el logro de las anteriores generaciones. Una desventaja de emplear regresiones del tipo de la ecuación (2) es que se dificulta hacer comparaciones en el tiempo o el espacio. Esto se debe a que la estimación del parámetro $\beta$ es sensible a la escala de la variable de interés. Una solución usada es re-escalar las variables de interés por la desviación estándar, lo cual tiene en cuenta las variaciones en el parámetro $\beta$ que son resultado de variaciones en la dispersión de $y_{t}$ o de $y_{t-1}$. Otra debilidad importante es que utiliza una relación lineal. En otras palabras, se parte del supuesto, por ejemplo, de que es lo mismo pasar de dos a tres años de educación que pasar de quince a dieciséis. La literatura sugiere que este supuesto no necesariamente se mantiene (Eide \& Showalter, 1999), sino que el grado de movilidad tiende a ser más elevado cuando se comparan poblaciones con bajos niveles educativos en relación con aquellas con más altos niveles educativos. Esta evidencia indica claramente heterogeneidad en la movilidad social intergeneracional.

\subsubsection{Regresión por cuantiles}

Para calcular el grado de movilidad en distintos puntos de la distribución, las regresiones son estimadas por cuantiles. En este caso, en vez de tener un indicador para la movilidad promedio, se tienen cálculos para diferentes niveles educativos o niveles de ingreso, según sea el caso. La técnica de regresión por cuantiles busca minimizar la suma de errores absolutos ponderados asimétricamente, para lo cual se usan los cuantiles seleccionados como estimadores de las ponderaciones.

En términos formales, para tener estimadores en varios puntos de la distribución, se fracciona la muestra en $n$ partes, según corresponda de acuerdo con el número de cuantiles a analizar. Siguiendo a Koenker y Bassett (1978), si se asume que $y_{t}(t=1, \ldots, T)$ es una variable aleatoria $Y$ con una función de distribución $F$, entonces el estimador $\beta$ del $\tau$-ésimo cuantil, se calcula como la solución al problema de optimización presentado en la ecuación (3). En este caso, se considera la suma ponderada del valor absoluto de los residuales de la ecuación de regresión de la siguiente forma: 


$$
\beta(\tau)=\underset{\beta \in R^{K}}{\arg \min } \frac{1}{T}\left[\sum_{t \in\left\{t: y_{t} \geq x_{t} \beta\right\}} \tau\left|y_{t}-x_{t} \beta\right|+\sum_{t \in\left\{t: y_{t} \geq x_{t} \beta\right\}}(1-\tau)\left|y_{t}-x_{t}^{\prime} \beta\right|\right]
$$

De donde se puede reformular el problema como:

$$
\min _{\beta \in R^{K}} \frac{1}{T} \sum_{t=1}^{T} \rho_{\tau}\left(y_{t}-X_{t}^{\prime}\right)
$$

La expresión en paréntesis no es más que el término de error, $\varepsilon$, y el ponderador $\rho_{\tau}$ viene dado por:

$$
\rho_{\tau}(\varepsilon)=\left\{\begin{array}{c}
\tau \varepsilon \text { si } \varepsilon \geq 0 \\
(1-\tau) \varepsilon \text { si } \varepsilon<0
\end{array}\right.
$$

En conclusión, el método consiste básicamente en estimar la regresión de la ecuación de transmisión intergeneracional de la educación o de los ingresos en diferentes puntos de la distribución.

\subsection{Desigualdad de oportunidades}

Ferreira y Gignoux (2008), basados en Roemer (1998), plantean un marco conceptual estilizado, con el fin de introducir su medida de desigualdad de oportunidades. La metodología de los autores parte de los siguientes planteamientos: se supone que y es la ventaja o "advantage" en el sentido de Roemer (1998), $C$ es un vector de variables de "circunstancias", ${ }^{3} E$ es un vector de variables de "esfuerzo"4 y $u$ son los efectos aleatorios o la suerte. Bajo el supuesto que los "esfuerzos" son endógenos y dependen de las "circunstancias", entonces la ventaja o resultado estará determinada como $y=f[C, E(C, v), u]$. Sobre esta base, la definición de igualdad de oportunidades de Roemer (1998) requiere que

2 El autor se refiere a la "igualdad de oportunidades" como una situación en la que los resultados importantes o "ventajas" se distribuyen independientemente de las circunstancias. Es decir, una situación donde la distribución del bienestar al interior de los grupos (cuyos integrantes comparten idénticas circunstancias) no cambia en estos grupos.

3 Factores que están fuera del alcance del individuo, donde no puede elegir.

4 Variables en las que el individuo puede elegir, por lo tanto tiene una "responsabilidad moral". 
la distribución condicional de la ventaja $(y)$ sea igual a la distribución no condicional, es decir, $F(t / C)=F(y)$ : cuanto más desigual sean las distribuciones, mayor será la desigualdad de oportunidades.

Fundamentados en lo anterior, Ferreira y Gignoux (2008) proponen una aproximación complementaria: crean índices de desigualdad de oportunidades de tipo escalar, al dividir a la población según las categorías de las variables circunstanciales. Tomando como base un vector de variables circunstanciales $C$, una partición de la distribución $\left\{y_{i}^{k}\right\}$ se define como aquella que $C_{i}^{k}=C^{k} \Leftrightarrow i \in k, k=1, \cdots, K$, siendo $K \leq N$ y $N$ el tamaño de la población. De esta manera, la población queda dividida en grupos cuyos miembros son idénticos respecto de las circunstancias del vector $C$. Para esto es necesario conocer el vector $C$, la distribución conjunta $F(y, C)$ y la división específica de cada variable. En ese sentido, los autores buscan una medida escalar $\theta:\left\{y_{i}^{k}\right\} \rightarrow \Re_{+}$que capture el grado de desigualdad de oportunidades en la división.

Ahora, sea $I B\left(\left\{y_{i}^{k}\right\}\right)$ el componente de desigualdad entre grupos en la división de la población realizada anteriormente, entonces la independencia estocástica implica que $F(y / C)=F(y) \Rightarrow I B\left(\left\{y_{i}^{k}\right\}\right)=0$. Dos candidatos para la medida escalar que se busca son los siguientes índices:

1. $\theta\left(\left\{y_{i}^{k}\right\}\right)=I B\left(\left\{y_{i}^{k}\right\}\right)$. En este caso, se trata de desigualdad de oportunidades absoluta, esto es el nivel de desigualdad entre los grupos de una población. Dichos grupos surgen de una división predeterminada de la población tal que los miembros de cada uno comparten las mismas circunstancias, en el sentido de Roemer (Ferreira \& Gignoux, 2008).

2. $\quad \theta\left(\left\{y_{i}^{k}\right\}\right)=I B\left(\left\{y_{i}^{k}\right\}\right) / I(F(y))$. Con esta alternativa, la desigualdad de oportunidades es relativa. Se supone la misma desigualdad entre los grupos, respecto de la desigualdad general en la población. Como cualquier medida relativa $\theta:\left\{y_{i}^{k}\right\} \rightarrow[0,1]$.

En términos prácticos, para obtener las medidas absolutas y relativas se calcula la desviación media logarítmica $(M L D$, por su sigla en inglés Mean Log Deviation), lo cual es básicamente un índice de entropía generalizado.

$$
M L D=\frac{1}{N} \sum_{i=1}^{N}\left(\ln \frac{\bar{y}}{y_{i}}\right)
$$

Para obtener el índice absoluto, se calcula la $M L D$ de la predicción de $y$ condicionada en los valores de las variables circunstanciales, es decir al vector $C$. 
El índice de desigualdad total no se detalla en el documento de Ferreira y Gignoux (2008), pero es posible deducirlo. Este índice es la MLD no condicionada, es decir, aplicada a la variable resultado o ventaja sin ningún tipo de controles que medien en esas desigualdades. Por lo tanto, es un indicador de la desigualdad en la variable de resultado que se está evaluando.

Luego de obtener los dos índices, absoluto y total, es posible calcular el índice de desigualdad relativo, que es simplemente la proporción de la desigualdad total que está representada por la desigualdad absoluta. Siendo así, a mayor índice relativo, mayor es el grado de desigualdad relacionado con las condiciones circunstanciales, sobre las cuales los individuos no tienen injerencia alguna. Visto de otra manera, cuando el valor del índice es alto, menor será el papel que pueden cumplir los esfuerzos propios de los individuos por alcanzar un mayor logro.

Posteriormente, Ferreira y Gignoux (2011) propusieron dos medidas relacionadas con la desigualdad educativa: una para los logros educativos y otra para las oportunidades educativas. La segunda es la de interés para este documento. En este sentido, plantean que la desigualdad de oportunidades educativas está dada por el porcentaje de la varianza en la variable resultado (en este caso los puntajes de la prueba PISA 2006), que se explica por las circunstancias predeterminadas. A continuación se resume el análisis.

Los autores plantean el siguiente índice como la medida de desigualdad de oportunidades (IOP) para la desigualdad entre tipos o grupos. Sea $C$ un vector de variables circunstanciales. La población puede estar dividida en grupos con circunstancias idénticas. Estas divisiones pueden estar agrupadas en tipos: $\Pi=$ $\left\{T_{1}, T_{2}, \ldots, T_{k}\right\}$, tal que $T_{1} \cup T_{2} \cup \ldots \cup T_{k}=\{1, \ldots, N\}, T_{1} \cap T_{k}=\varnothing, \forall l, k, y$ los vectores $C i=C j, \forall i, j \mid i \in e T k, j \in T k, \forall k$.

El índice será $\theta_{I O P}=I\left(\left\{u_{i}^{k}\right\}\right) / I(y)^{\prime}$ donde $\left\{u_{i}^{k}\right\}$ es la distribución suavizada correspondiente a la distribución $y$, y a la división $\Pi$.

Este índice "puede ser calculado de forma no-paramétrica por medio de una descomposición estándar de la desigualdad entre grupos" (Ferreira \& Gignoux, 2011, p. 16). Sin embargo, este procedimiento requiere de grandes bases de datos cuando el vector C es grande. Siguiendo a Bourguignon et al. (2007), Ferreira y Gignoux (2008) proponen una alternativa paramétrica, basados en una regresión por $\mathrm{MCO}$ :

$$
\hat{\theta}_{I O P}=\frac{I\left(C_{i}^{\prime} \hat{\beta}\right)}{I(y)}
$$


Donde $C_{i}^{\prime} \hat{\beta}$ representa el vector de la variable resultado predicha, obtenida de la regresión $y_{i}=C_{i}^{\prime} \hat{\beta}+\eta_{i}$. Bajo el supuesto de una relación lineal entre la variable resultado y las circunstancias, este vector es equivalente a la distribución suavizada, ya que todos los individuos con idénticas circunstancias son asignados a sus valores medios condicionales.

En una modificación a la ecuación (7), Ferreira y Gignoux (2011) utilizan la varianza simple como su índice de desigualdad $I()$ :

$$
\hat{\theta}_{I O P}=\frac{\operatorname{Var}\left(C_{i}^{\prime} \hat{\beta}\right)}{\operatorname{Var}\left(y_{i}\right)}
$$

Este índice tiene cuatro características atractivas:

1. Simplicidad de cálculo, pues es equivalente al $R^{2}$ de una regresión lineal simple de la variable dependiente sobre un vector $C$ de circunstancias individuales.

2. Permite obtener un resumen estadístico significativo, al ser una aproximación paramétrica del límite inferior de la proporción de la desigualdad general en la variable resultado, que se explica causalmente por circunstancias predeterminadas. Se dice que es un límite inferior, porque, al disponer de un vector $C$ de circunstancias más comprehensivo, la proporción de la desigualdad total puede ser mayor, ya que en ese caso al tener más variables incluidas en la regresión, el $R^{2}$ tiende a aumentar.

3. “Permite el uso de más información sobre las circunstancias que los estudios anteriores, los cuales se basan, por lo general, en un conjunto más pequeño de variables de fondo, y por lo tanto capturan una parte más limitada de la heterogeneidad en los recursos familiares" (Ferreira \& Gignoux, 2011, p. 18).

4. Este es un estimador paramétrico de una relación $\left(\theta_{I O P}=I\left(\left\{u_{i}^{k}\right\}\right) / I(y)\right)$ que es invariante (en términos cardinales) a la estandarización de la variable de resultado.

5. Es posible descomponerlo perfectamente en componentes para cada variable individual en el vector $C$. Así la ecuación (8) puede reescribirse como:

$$
\hat{\theta}_{I O P}=(\operatorname{var} y)^{-1}\left[\sum_{j} \beta_{j}^{2} \operatorname{var} C_{j}+\frac{1}{2} \sum_{k} \sum_{j} \beta_{k} \beta_{j} \operatorname{cov}\left(C_{k}, C_{j}\right)\right]
$$


Este, a su vez puede ser escrito como la suma de todos los elementos (denotados por $j$ ) del vector $C$ :

$$
\hat{\theta}_{I O P}=\sum_{j} \hat{\theta}^{j}=\sum_{j}(\operatorname{var} y)^{-1}\left[\beta_{j}^{2} \operatorname{var} C_{j}+\frac{1}{2} \sum_{k} \beta_{k} \beta_{j} \operatorname{cov}\left(C_{k}, C_{j}\right)\right]
$$

6. Finalmente, el índice también puede ser visto como isomorfo a una medida de la persistencia intergeneracional de la desigualdad, lo inverso de una medida de movilidad. Tomando la regresión de Galton del resultado del hijo $\left(y_{t}\right)$ sobre el resultado del padre $\left(y_{t-1}\right)$ :

$$
y_{t}=\beta y_{t-1}+\varepsilon
$$

En este caso, el coeficiente $\beta$ es usado como una medida de persistencia y 1 - $\beta$ como medida de movilidad — también llamada índice gamma- Si se reemplaza $y_{t-1}$ con un vector de variables de características de los padres o la familia, la ecuación (11) se transformaría en algo similar a $y_{i}=C_{i}^{\prime} \hat{\beta}+\eta_{i^{\prime}}$ y el $R^{2}$, medida de inmovilidad, sería la medida de desigualdad de oportunidades $\left(\hat{\theta}_{I O P}\right)^{\prime}$. Así, $\hat{\theta}_{I O P}$ es "una medida de persistencia intergeneracional o inmovilidad, en la cual los valores faltantes de la variable resultado de los padres, $y_{t-1}$, se reemplaza por un vector proxy del background circunstancial familiar, $C_{i}^{\prime \prime}$ (Ferreira \& Gignoux, 2011, p. 19).

De acuerdo con lo planteado por Ferreira y Gignoux (2011), el índice tomará valores entre 0 y 1 , en donde un índice más cercano a uno indica mayor desigualdad. Nótese, sin embargo, que este debe interpretarse como un límite inferior de la proporción de la variación total en la variable resultado que se explica por circunstancias predeterminadas (por ejemplo, género y antecedentes familiares). Con ello, un índice de desigualdad relativo del 30\%, estaría indicando que al menos un $30 \%$ de la desigualdad está explicado por circunstancias propias de la persona al nacer o que no dependen de su esfuerzo propio. En otras palabras, la desigualdad de oportunidades da cuenta de por lo menos $30 \%$ de la desigualdad prevaleciente en la variable de resultado analizada.

\subsection{Medición de la calidad de vida}

La medición de la pobreza o la calidad de vida es una tarea compleja. Dentro de las alternativas, se han planteado diversas opciones, como son las líneas de pobreza, el índice de Necesidades Básicas Insatisfechas (NBI), el Índice de 
Calidad de Vida (ICV), los puntajes SISBEN, entre otros. Dado que el puntaje SIBEN no está disponible y que los otros índices - ICV y NBI- solo se encuentran a nivel agregado, en este trabajo se propone el cálculo de un Índice de Condiciones Materiales-ICM a nivel de hogar, para obtener una medición con microdatos de la situación de movilidad en torno de las condiciones materiales de los individuos y los hogares.

Para obtener el ICM se adapta la metodología empleada en el cálculo del puntaje del SISBEN III. Este último se fundamenta en la definición del enfoque de realizaciones y capacidades de Sen (1998), basándose en lo que el individuo es y puede hacer con los bienes que posee, así como de sus características personales y el entorno que lo rodea. También tiene en cuenta el conjunto de todos los posibles funcionamientos que el individuo es capaz de alcanzar (oportunidades reales, las posibilidades de opción para elegir, entre otras). Asimismo, el enfoque permite hacer comparaciones interpersonales, ya que se conforma un patrón común donde se evalúan las condiciones de vida de los hogares. En este sentido, es una medida más robusta que, por ejemplo el NBI, pues con este último un par de hogares que tengan una necesidad básica insatisfecha como la vivienda inadecuada, es contada como pobre, de la misma manera que los que presentan deficiencias por inasistencia escolar. El SISBEN III le da ponderaciones a las dimensiones consideradas para catalogar a un hogar o individuo como pobre de acuerdo con lo que es la norma en el contexto analizado. De esta manera, en las áreas rurales el poseer viviendas hechas en madera, si son la norma, no conllevan a categorizar a un hogar como pobre.

Para la aplicación del punto de vista de Sen (1998) sobre capacidades, es necesario identificar el conjunto de dimensiones consideradas socialmente primordiales. En el caso del SISBEN III, el conjunto de dimensiones identificadas corresponde a las variables relacionadas con las características de la vivienda; del individuo (como la salud y la educación); y del contexto social y del hogar (como son la vulnerabilidad individual y la contextual) según se detalla en la tabla 1.

Una vez se identifican las dimensiones primordiales, se procede a calcular el índice agregado. Para ello es necesario definir las ponderaciones para las categorías existentes al interior de cada dimensión ${ }^{5}$ y definir ponderaciones para cada una de las dimensiones. El método estadístico empleado para calcular el índice es el de conjuntos borrosos (Lelli, 2001), que se detalla a continuación.

5 Esto es, se debe obtener una ponderación para cada una de las opciones presentes en, por ejemplo, los materiales del piso, a saber: mármol, baldosas, madera, cemento, arena. 
Tabla 1. Componentes del índice SISBEN III

\begin{tabular}{lccl}
\hline \multicolumn{1}{c}{ Vivienda } & Salud & Educación & \multicolumn{1}{c}{ Vulnerabilidad } \\
\hline - Tipo de unidad de & - Discapacidad & - Porcentaje de & Individual: \\
vivienda. & permanente & adultos con & - Número de personas \\
- Fuente de agua & - Presencia de & analfabetismo & en el hogar. \\
para consumo. & adolescentes con & funcional. & - Tipo de jefatura. \\
- Tipo de conexión & hijos. & - Porcentaje de ina- & - Tasa de dependencia \\
sanitaria. & & sistencia escolar. & demográfica. \\
- Exclusividad del & & Atraso escolar. & - Tenencia de activos. \\
sanitario. & - Porcentaje de & \\
- Material de los & & niños trabajando. & Contextual: \\
pisos. & - Porcentaje de & - Tasa de mortalidad \\
- Material de las & niños con secun- & infantil. \\
paredes. & daria incompleta & - Tasa de homicidios. \\
- Eliminación de & o menos. & - Tasa de cobertura neta \\
basuras. & & por nivel educativo. \\
- Tipo de combusti- & & & Tasa de uso de servi- \\
ble para cocinar. & & & cios de salud general \\
- Hacinamiento. & & dada una necesidad. \\
\hline
\end{tabular}

Fuente: elaboración de los autores con base en Flórez et al. (2008).

Nota: para los cálculos del índice con encuestas diferentes a las del SISBEN, solo se logra una aproximación, pues existen variables como la discapacidad permanente o el analfabetismo funcional que regularmente no se encuentran en los formularios de las encuestas de hogares o las de calidad de vida.

Formalmente, sea $\mathrm{A}$ un conjunto dado para el cual se define un subconjunto borroso de este como $\mathrm{B}=\left\{a, \mu_{B}(a) \mid a \in A^{\wedge} \mu_{B}(a): A \rightarrow[0,1]\right\}, y \mu_{B}(a)$ es la función que mide el grado de pertenencia de $a$ al conjunto B. En el SISBEN III, por ejemplo, $A$ puede representar un conjunto de $n$ hogares y $B$ el subconjunto difuso de la población que reside en una vivienda que no cumple las condiciones mínimas de habitabilidad. El grado de pertenencia a un conjunto es una función que puede tomar valores entre cero y uno. La función de pertenencia se definiría como:

$\mu_{B}(a)=0$ si un hogar $a$ no pertenece al subconjunto B.

$\mu_{B}(a) \in(0,1)$ si el hogar $a$ pertenece de manera parcial al subconjunto $B$.

$\mu_{B}(a)=1$ si el hogar $a$ pertenece totalmente al subconjunto B.

La especificación de la función de pertenencia es la definida por Cheli y Lemmi (1995), la cual evita definiciones de umbrales de forma arbitraria. Según esta especificación, el grado de pertenencia de un individuo es definido de acuerdo con la posición ocupada de este en el total de la población. De este modo, la función de pertenencia vendría dada por: 


$$
\begin{gathered}
0 \text { si } a=a^{(1)} ; k=1 \\
\mu_{B}(a)=\left\{\mu_{B}\left(a^{(k-1)}\right)+\frac{F\left(a^{(k)}\right)-F\left(a^{(k-1)}\right)}{1-F\left(a^{(1)}\right)} \text { si } a=a^{(k)} ; k>1\right. \\
1 \text { si } a=a^{(k)} ; k=K
\end{gathered}
$$

Donde $F($.) es la distribución acumulada de $a$. Asimismo, $k$ está asociado con el riesgo de privación, el cual se encuentra ordenado de tal manera que $k=1, \ldots$, $K$, siendo 1 el nivel más bajo de privación y K el más alto, cuando se trata de un índice de pobreza, como es el índice SISBEN. Para los efectos de esta investigación, el cálculo del índice reflejará el estándar de vida, o la calidad de vida, con lo cual $K$ representa el menor nivel de privación.

Finalmente, se define $W_{j}$ como la ponderación de las funciones que miden el grado de pertenencia a cada dimensión $j$, tal que:

$$
W_{j}=-\ln \left(\frac{\sum_{a=1}^{n} \mu_{j}(a)}{n}\right)
$$

Teniendo las ponderaciones de las dimensiones consideradas, el puntaje del ICM se calcula como la suma ponderada de todas las dimensiones $T$, para cada hogar $i$.

$$
I C M_{i}=\frac{\sum_{j=1}^{T} \mu_{j}(a) W_{j}}{\sum_{j=1}^{T} W_{j}}
$$

Una aplicación de esta metodología es llevada a cabo para construir un índice de calidad del empleo para el mercado laboral de Chile (Gómez et al., 2013).

\section{Resultados}

En este apartado se muestran los índices de movilidad social. En primer lugar se calculan los índices promedio de movilidad intergeneracional en educación y se relacionan con las condiciones de pobreza y desigualdad a nivel regional, para el grupo de edad entre 25 y 65 años. En seguida, se presentan los índices 
de movilidad por cuantiles, según los años de educación. En esta primera parte se emplean las ECV de 2003 y 2010. Luego, se calcula el ICM y se lleva a cabo el análisis de la movilidad en este índice, al emplear la ECVMS de 2010. ${ }^{6}$ Finalmente, se analiza la relación entre la movilidad y la desigualdad de oportunidades, siguiendo la propuesta de Ferreira y Gignoux (2011).

\section{1 Índices de movilidad intergeneracional promedios en educación}

En primer lugar, se presentan los resultados promedio, obtenidos a partir de la ecuación de movilidad intergeneracional en educación. En este caso se emplean dichos promedios para compararlos con condiciones de desigualdad en el ingreso regional.

Al calcular los índices de movilidad por regiones, se encuentran altos índices de movilidad intergeneracional en San Andrés y Bogotá. La región Pacífica por su parte muestra los índices más bajos de movilidad (tabla 2).

Tabla 2. Índices de movilidad intergeneracional por regiones, 2010

\begin{tabular}{lc}
\hline \multicolumn{1}{c}{ Región } & $\begin{array}{c}\text { Movilidad intergeneracional } \\
\text { (Índice gamma) }\end{array}$ \\
\hline San Andrés y Providencia & 0,74 \\
Bogotá D.C. & 0,62 \\
Orinoquía-Amazonía & 0,60 \\
Valle & 0,60 \\
Antioquia & 0,57 \\
Caribe & 0,52 \\
Central & 0,49 \\
Oriental & 0,46 \\
Pacífica (Sin Valle) & 0,36 \\
\hline
\end{tabular}

Fuente: cálculos de los autores.

En la figura 1 se muestra la relación positiva entre el índice de movilidad intergeneracional en educación y el PIB per cápita por regiones, cuya correlación es de 0,54. En este caso, se evidencia que la movilidad en educación es mayor en las zonas más prósperas. San Andrés es un punto atípico en este sentido, pues a pesar de pertenecer a una región bastante rezagada, como lo es el Caribe,

6 En los anexos 1 y 2 se presentan las estadísticas descriptivas de los datos empleados en esta sección. 
presenta los índices de movilidad más altos. Por lo anterior, en lo subsiguientes análisis se agregará San Andrés al resto de la región Caribe, obteniéndose una muestra más representativa para el análisis.

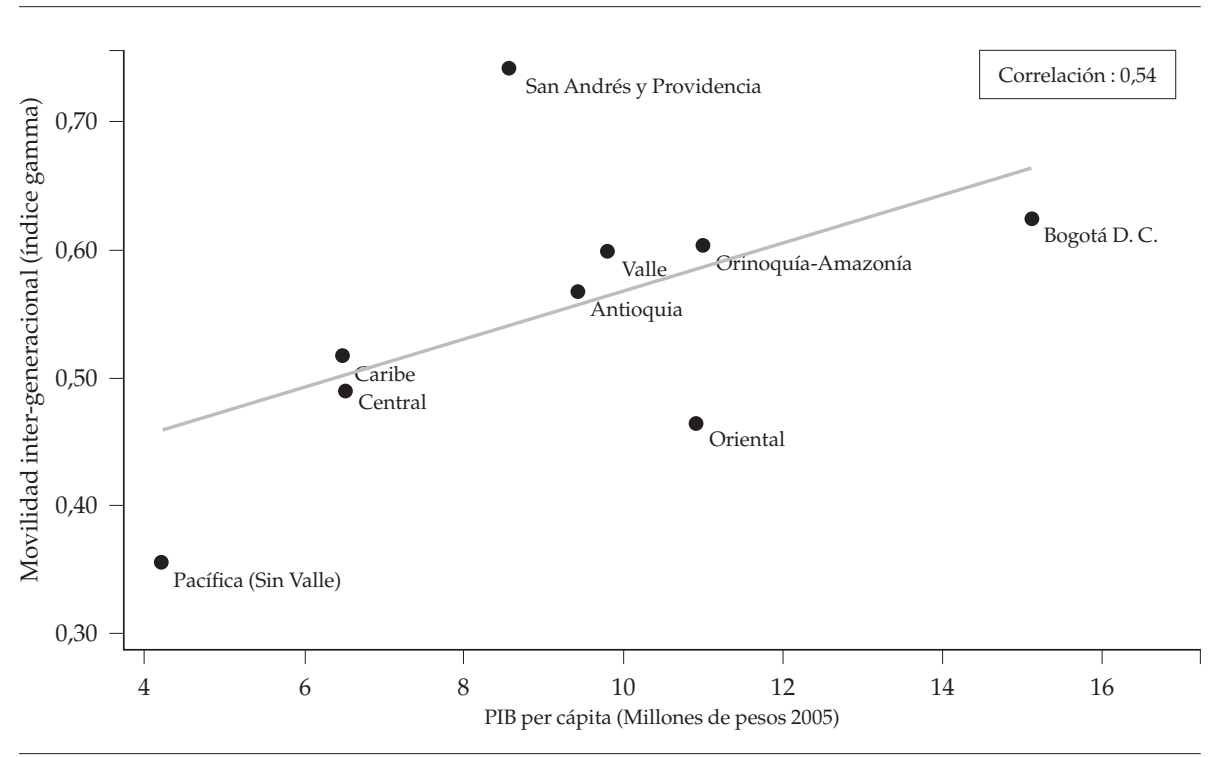

Figura 1. Relación entre la movilidad y el PIB per cápita, 2010

Fuente: cálculos de los autores.

Nota: Las regiones están compuestas de la siguiente forma. Pacífica: Chocó, Cauca y Nariño. Caribe: Guajira, Cesar, Magdalena, Atlántico, Bolívar, Sucre y Córdoba. Oriental: Norte de Santander, Santander, Boyacá, Cundinamarca y Meta. Central: Caldas, Quindío, Risaralda, Tolima, Huila y Caquetá. Orinoquía-Amazonía: Arauca, Casanare, Vichada, Guainía, Guaviare, Vaupés, Amazonas y Putumayo.

Asimismo, se puede mencionar que las regiones más pobres, además de tener altas desigualdades en el ingreso, a juzgar por el índice de Gini, también exhiben los menores índices de movilidad intergeneracional (figura 2).

\section{2 Índices de movilidad social en educación}

En este apartado se presentan los resultados de las estimaciones de la ecuación de movilidad intergeneracional en educación por el método de regresión por cuantiles. En dicha ecuación, la variable dependiente corresponde a los años de educación de los hijos, en relación con el promedio de los años alcanzados por los otros individuos de similar edad. La variable independiente corresponde al nivel educativo de la madre. Normalizar por el promedio de años de educación 


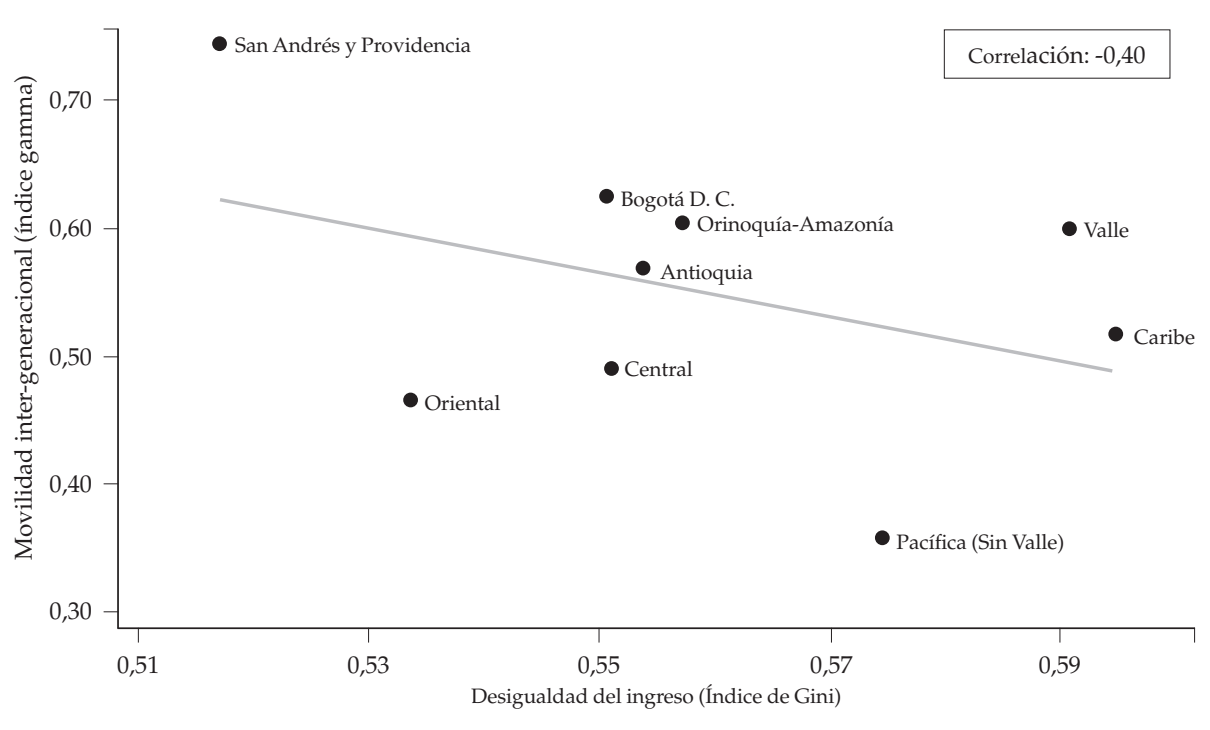

Figura 2. Movilidad intergeneracional contra desigualdad regional, 2010

Fuente: cálculos de los autores.

Nota: las regiones están compuestas así. Pacífica: Chocó, Cauca y Nariño. Caribe: Guajira, Cesar, Magdalena, Atlántico, Bolívar, Sucre y Córdoba. Oriental: Norte de Santander, Santander, Boyacá, Cundinamarca y Meta. Central: Caldas, Quindío, Risaralda, Tolima, Huila y Caquetá. Orinoquía-Amazonía: Arauca, Casanare, Vichada, Guainía, Guaviare, Vaupés, Amazonas y Putumayo.

alcanzados por la cohorte de edad en la que se encuentran los individuos ayuda a controlar por los diferenciales que provienen del ciclo de vida. Esto es, individuos más jóvenes pueden estar por debajo del nivel educativo de los padres porque no han avanzado en su ciclo educativo.

En general, los resultados del análisis muestran que las regiones de Colombia tienen bajos niveles de movilidad intergeneracional en educación. No obstante, comparando los cálculos de 2003 con los de 2010 se encuentra que ha habido mejoras en este sentido.

En primer lugar, se presentan resultados que permiten hacer la comparación con ciertas características de los individuos que son ajenas a su voluntad, como el género y la condición étnica. En relación con el género, la movilidad intergeneracional de ambos sexos mejoró en 2010; no obstante, las diferencias entre estos se mantienen relativamente imperceptibles. Estos resultados son compatibles con la evidencia mostrada por algunos trabajos empíricos existentes en Colombia (Gaviria, 2002; Angulo et al., 2012). 

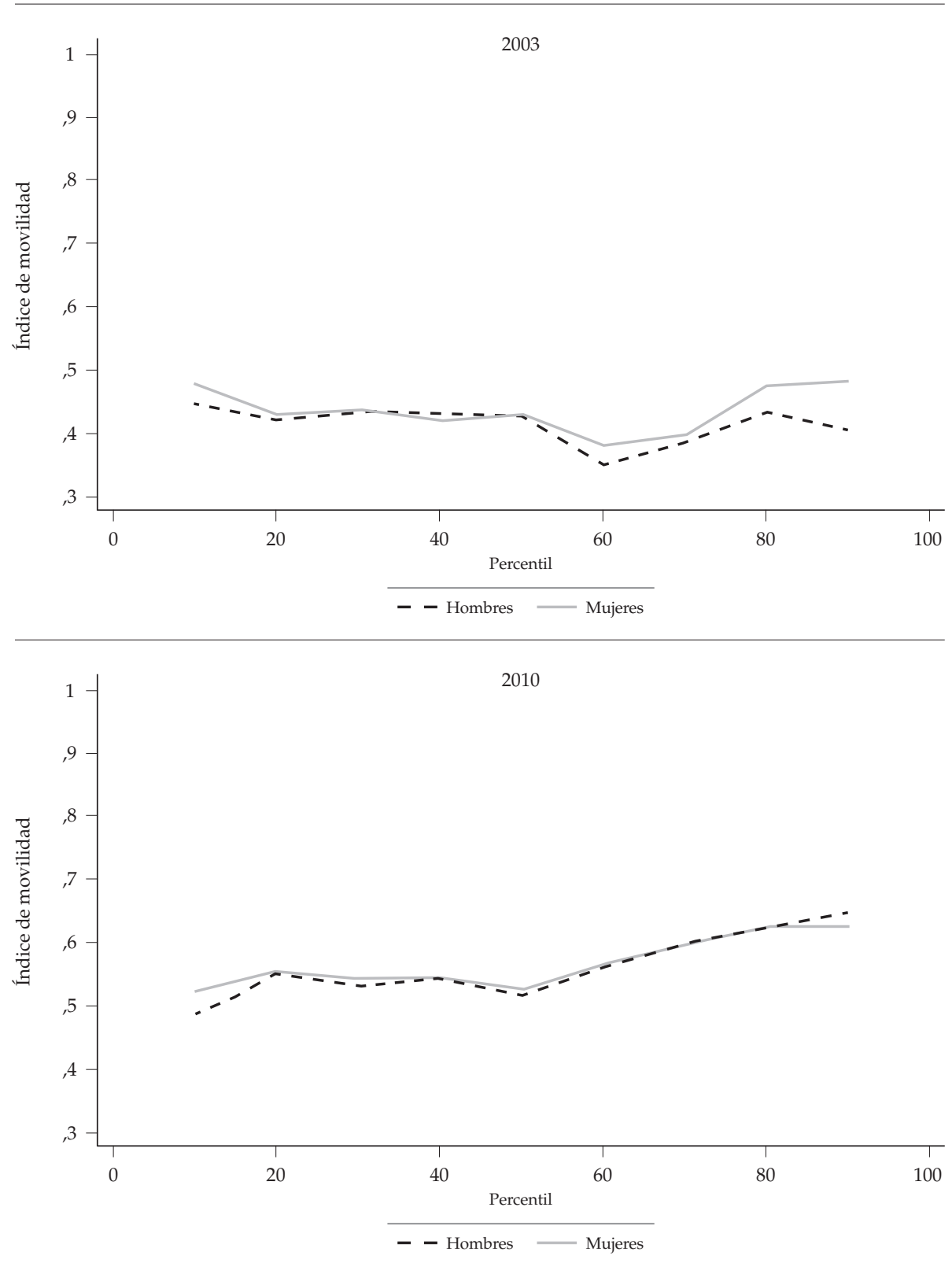

Figura 3. Índice de movilidad, según género por percentiles del nivel educativo, 2003-2010

Fuente: cálculos de los autores.

Otra característica que reviste interés para el análisis de la movilidad social es la condición étnica. De acuerdo con tal clasificación, los resultados demues- 
tran la presencia de diferencias significativas. Según se presenta en la figura 4, los indígenas son los que logran menores índices de movilidad. Esto sugeriría
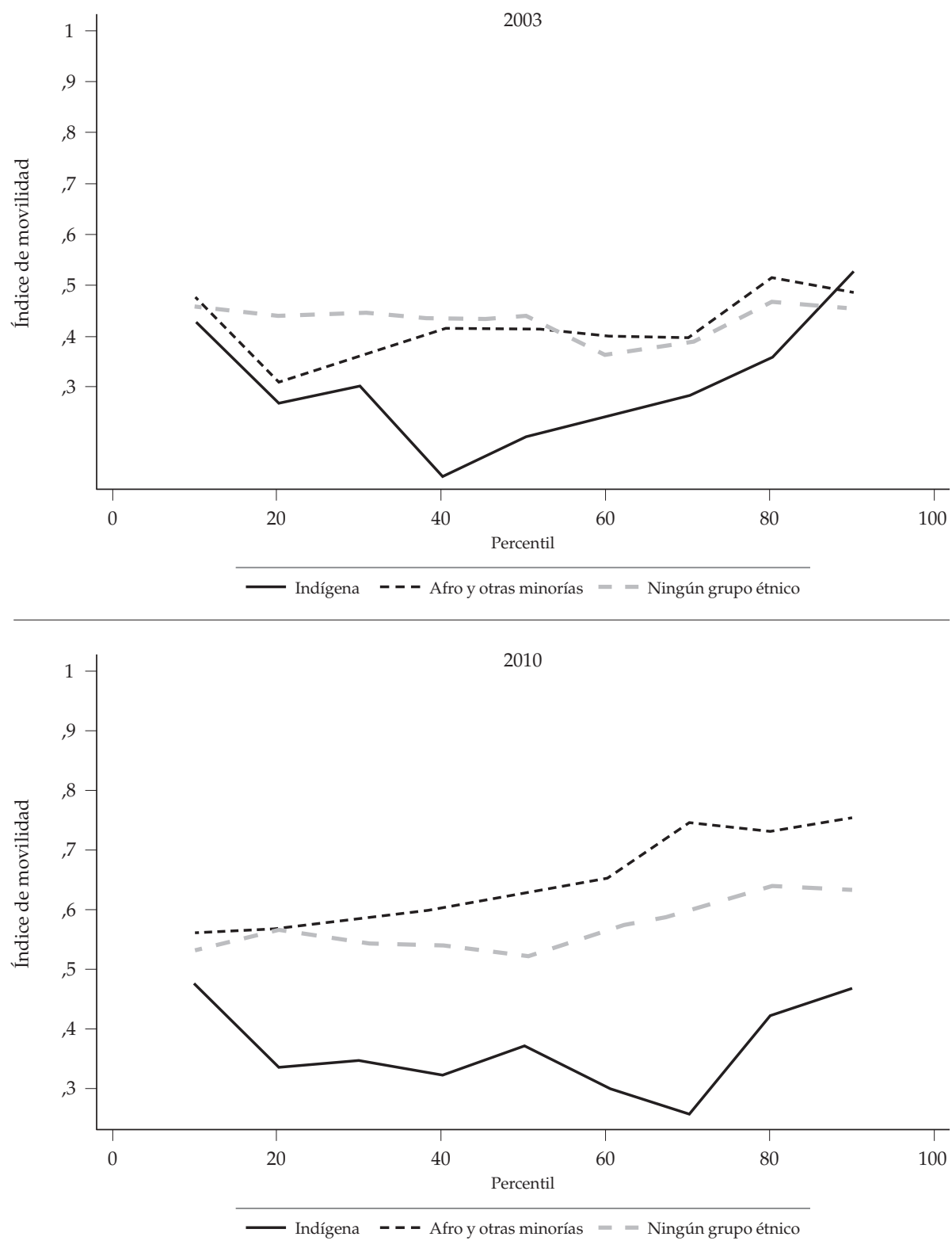

Figura 4. Índice de movilidad intergeneracional, según condición étnica por percentiles del nivel educativo, 2003-2010

Fuente: cálculos de los autores. 
que la situación experimentada por los indígenas se caracteriza por la existencia de condiciones de persistencia en la situación que presentaban los padres. Se puede notar también que los demás grupos étnicos presentan ganancias en la movilidad que no son observadas por los indígenas, si se compara 2003 con 2010, ya que en este último año los demás grupos étnicos alcanzan una movilidad que está muy por encima de los que logran los indígenas.

La figura 5 muestra los índices de movilidad por cuantiles, calculados a partir de la ecuación de movilidad intergeneracional en educación. Se puede
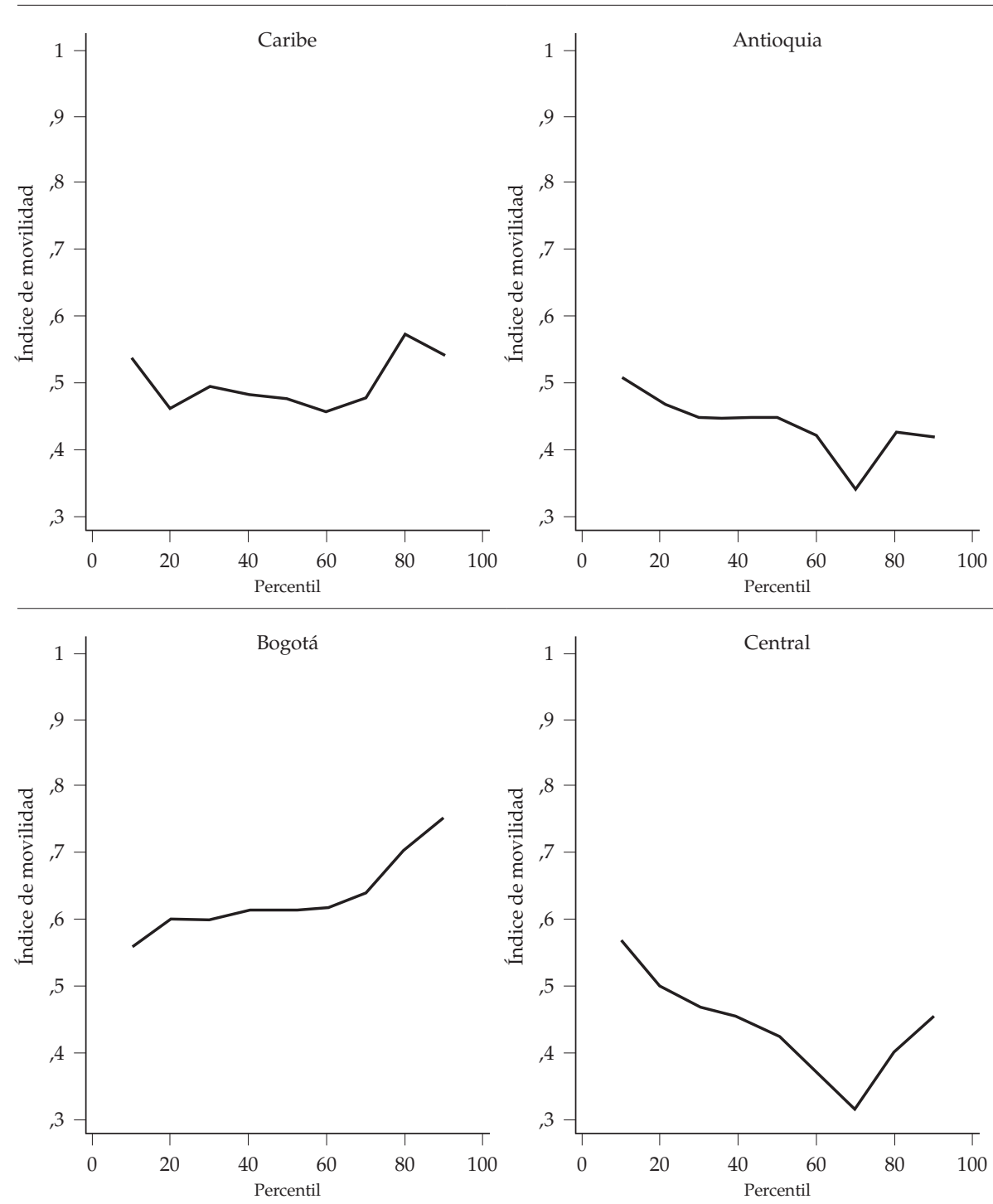

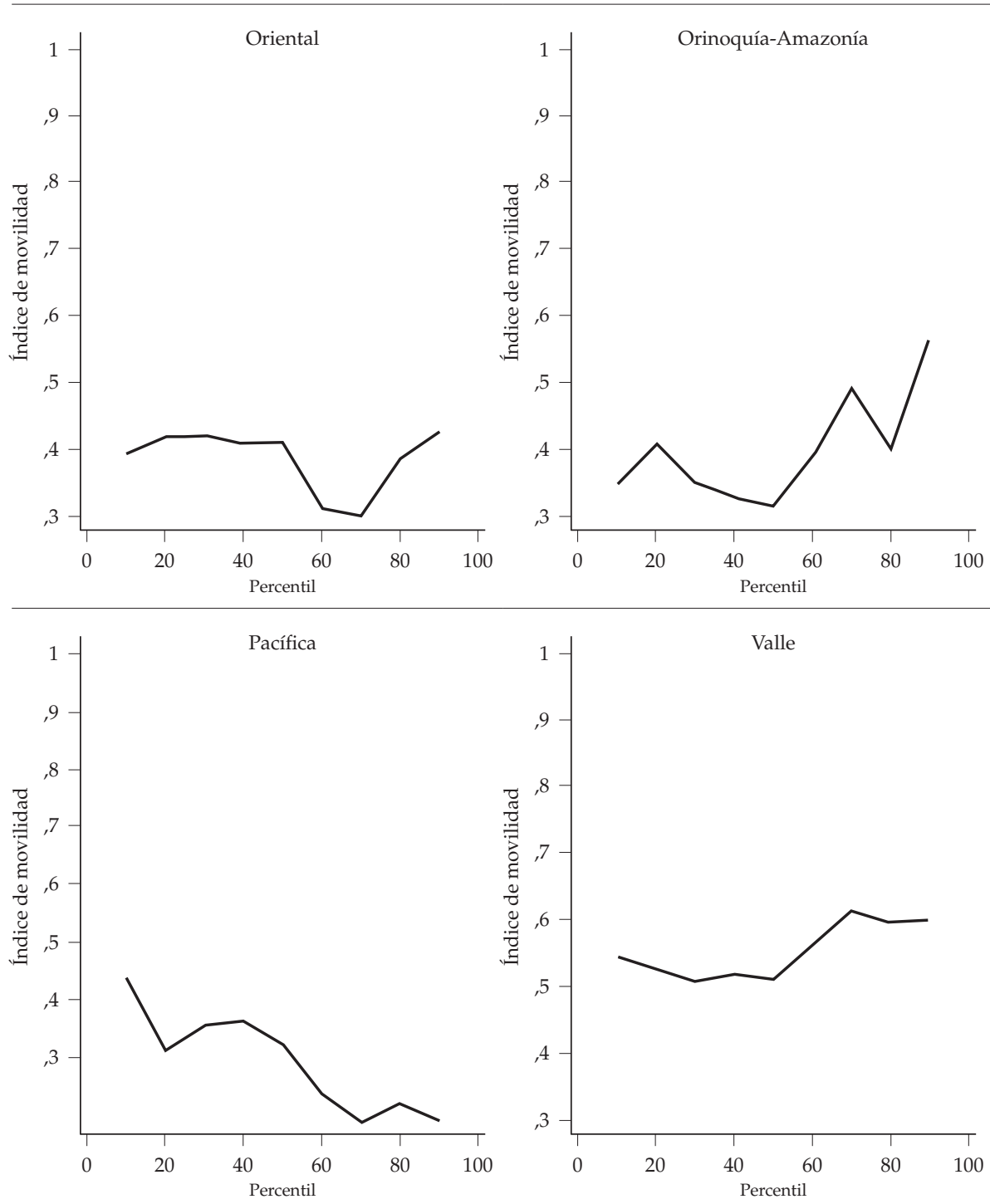

Figura 5. Índice de movilidad según regiones por percentiles del nivel educativo, 2003 Fuente: cálculos de los autores.

observar que los resultados no son homogéneos ni por cuantiles, ni por regiones. En general, mayor grado de educación está asociado con mayor movilidad. Es decir, que quienes se ubican en los últimos cuantiles de la distribución son los que logran alcanzar mayor movilidad. Esta característica agrava la situación en relación con las desigualdades, pues los que están en una mejor posición son los que logran mejorar más en términos de movilidad. 
En cuanto a las regiones, Bogotá es la ciudad que presenta los mayores índices de movilidad intergeneracional, con cifras que se ubican en 0,55 y 0,75. En la capital, los índices de movilidad van aumentando gradualmente con el nivel educativo, con lo cual podría decirse que es en esta ciudad donde se logra romper en mayor grado el círculo de la persistencia en la transmisión intergeneracional de la educación. Por otro lado, las regiones Pacífica, Oriental, Orinoquía y Antioquia son las que menor movilidad presentan. En el Pacífico, los índices no alcanzan 0,5 y van reduciéndose a medida que avanzamos en el nivel educativo.

En relación con los resultados para el año 2010, lo que salta a la vista es que en general las curvas se ubican en niveles más altos respecto de los encontrados para 2003. Esto quiere decir que en relación con dicho año, en 2010 se han presentado mejorías en los índices de movilidad social. Este resultado es relativamente consistente en todas las regiones, con lo cual las ganancias en movilidad, podría decirse, son generalizadas en todo el país. La Costa Pacífica y la Región Oriental son las que menos se destacan en este sentido, es decir, las que menores índices de movilidad presentan. Antioquia, por su parte, dejó de estar entre el grupo de los menos destacados.

Ahora bien, nuevamente Bogotá es la que muestra el mejor desempeño en este indicador, no solamente porque es la que mayor movilidad presenta, sino porque es la que mayores ganancias muestra. También se nota que en el Pacífico los mayores niveles de educación están asociados con menor movilidad. Esto puede ser el resultado de varios elementos, como por ejemplo, mejor infraestructura educativa en la capital, mejor calidad de los profesores, mayores tasas de retención en el sistema escolar, o simplemente, mayor inclinación de los padres porque sus hijos logren mejorías en su calidad de vida, al invertir más decisivamente en sus hijos. De todas formas, es muy complicado descifrar los factores que explican este resultado y ese no es el objetivo del presente trabajo. No obstante lo anterior, algo en lo que sí se avanza en esta investigación es en la relación con la movilidad social y la desigualdad de oportunidades, factor que también puede ser determinante de una situación como la que se encuentra en la capital del país.

\section{3 Índices de movilidad en calidad de vida}

Otras formas empleadas para medir la movilidad social, alternativas a la movilidad intergeneracional en educación, son el uso de indicadores socioeconómicos como ingresos o calidad de vida. En este apartado también se ha calculado la movilidad social, al aplicar matrices de transición a un índice de calidad de vida, obtenido a partir de la formulación empleada para los índices de pobreza del SISBEN III. Para este efecto, se utilizó la ECVMS, la cual realiza una serie de 
preguntas retrospectivas para evaluar las condiciones socioeconómicas de los individuos cuando estos tenían diez años de edad, que reflejarían las condiciones de los padres. A partir de estas características se calcula el Índice de Condiciones Materiales (ICM) de los padres o de antecedentes familiares, que luego es comparado con el índice calculado para las condiciones actuales y permite conocer cuál ha sido la movilidad socioeconómica de los individuos.
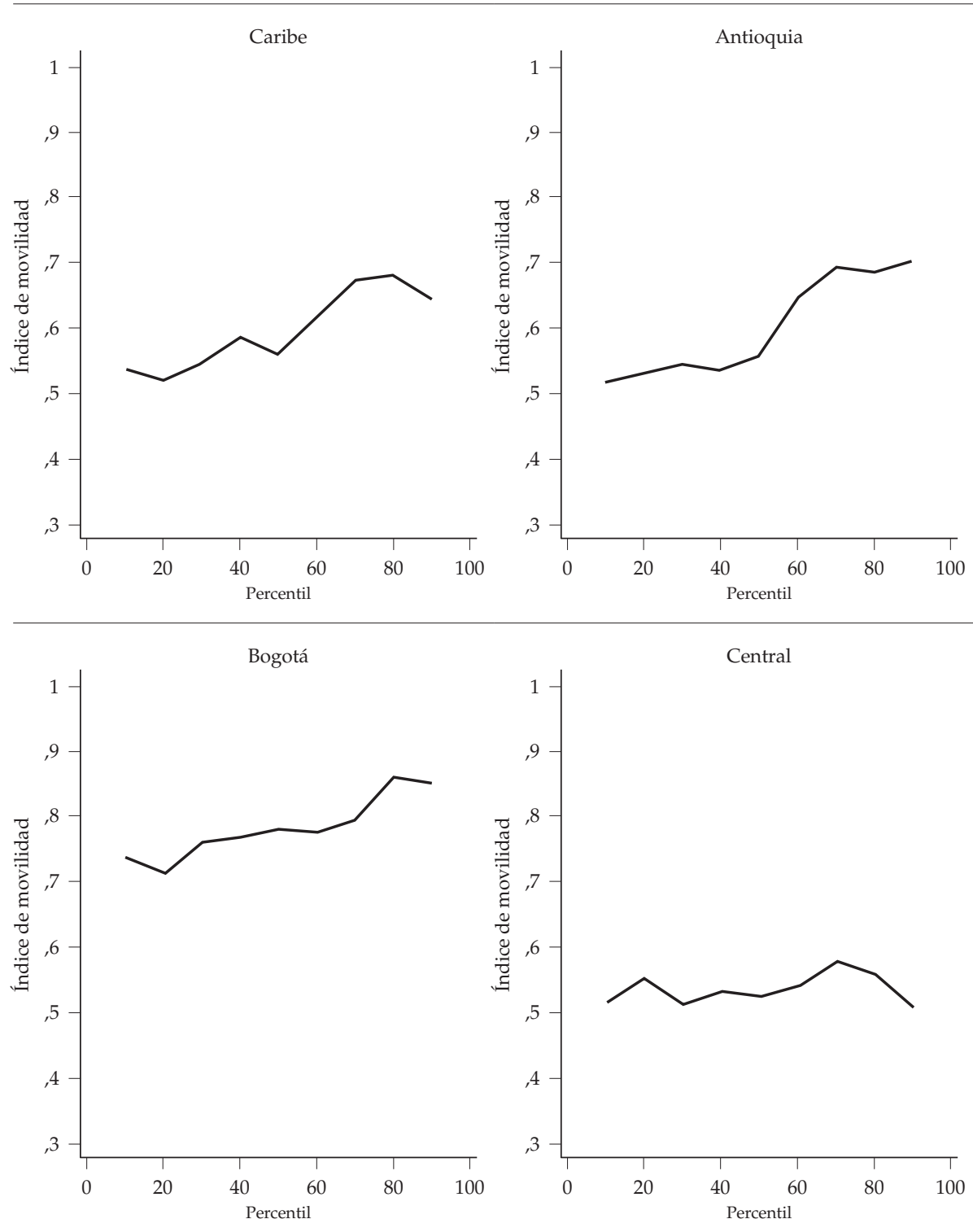

Continuación figura 6 

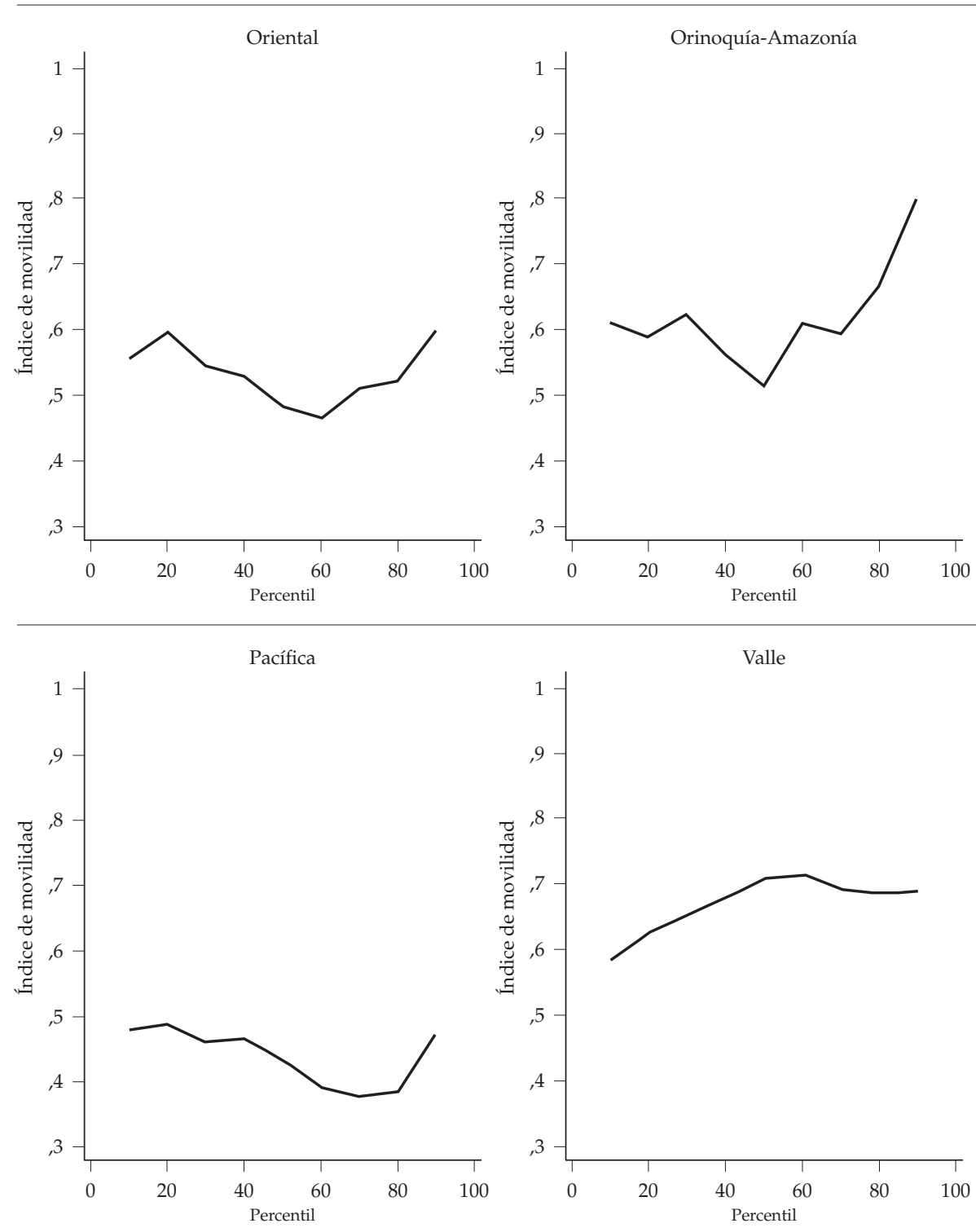

Figura 6. Índice de movilidad según regiones por cuantiles por percentiles del nivel educativo, 2010

Fuente: cálculos de los autores.

Para la construcción del ICM se emplearon las preguntas retrospectivas y las condiciones actuales, al hacer uso de las condiciones socioeconómicas que se presentan en la tabla 3. Algunos atributos que se tienen en cuenta en las preguntas 
retrospectivas cambian respecto de la situación actual, por ejemplo, en el pasado la presencia del servicio de parabólica o de Internet no era tan generalizado.

Tabla 3. Atributos socioeconómicos de las condiciones retrospectivas y actuales usados para el ICM, 2010

\begin{tabular}{|c|c|}
\hline $\begin{array}{l}\text { Bienes que poseía el individuo en su hogar } \\
\text { (en el pasado) }\end{array}$ & $\begin{array}{l}\text { Bienes que posee el individuo en su hogar } \\
\text { (en la actualidad) }\end{array}$ \\
\hline Lavadora & Lavadora \\
\hline Nevera & Nevera \\
\hline Licuadora & Licuadora \\
\hline Estufa & Estufa \\
\hline Plancha & Plancha \\
\hline Horno eléctrico & Horno eléctrico \\
\hline TV a color & TV a color \\
\hline Equipo de sonido & Equipo de sonido \\
\hline Aspiradora & Aspiradora \\
\hline Aire acondicionado & Aire acondicionado \\
\hline Ventilador & Ventilador \\
\hline Auto & Auto \\
\hline Casa de recreo & Casa de recreo \\
\hline \multirow[t]{6}{*}{ Bicicleta } & Moto \\
\hline & Horno microondas \\
\hline & Calentador \\
\hline & Computador \\
\hline & Parabólica \\
\hline & Internet \\
\hline Sitio de donde obtenían el agua & Sitio de donde obtenían el agua \\
\hline Tipo de servicio sanitario (inodoro, letrina...) & Tipo de servicio sanitario \\
\hline Material predominante de los pisos & Material predominante de los pisos \\
\hline
\end{tabular}

Fuente: elaboración de los autores con base en la ECVMS.

Como se observa en la figura 7, la situación de los hogares ha presentado mejoras en la distribución de los indicadores de calidad de vida. Se puede notar que la media de la distribución se desplaza hacia la derecha, donde las condiciones de los individuos son más favorables en tanto que el índice ponderado 
de calidad de vida alcanza cifras más altas. Ello es el reflejo de la presencia de atributos materiales en el hogar que mejoran la calidad de vida de los individuos.

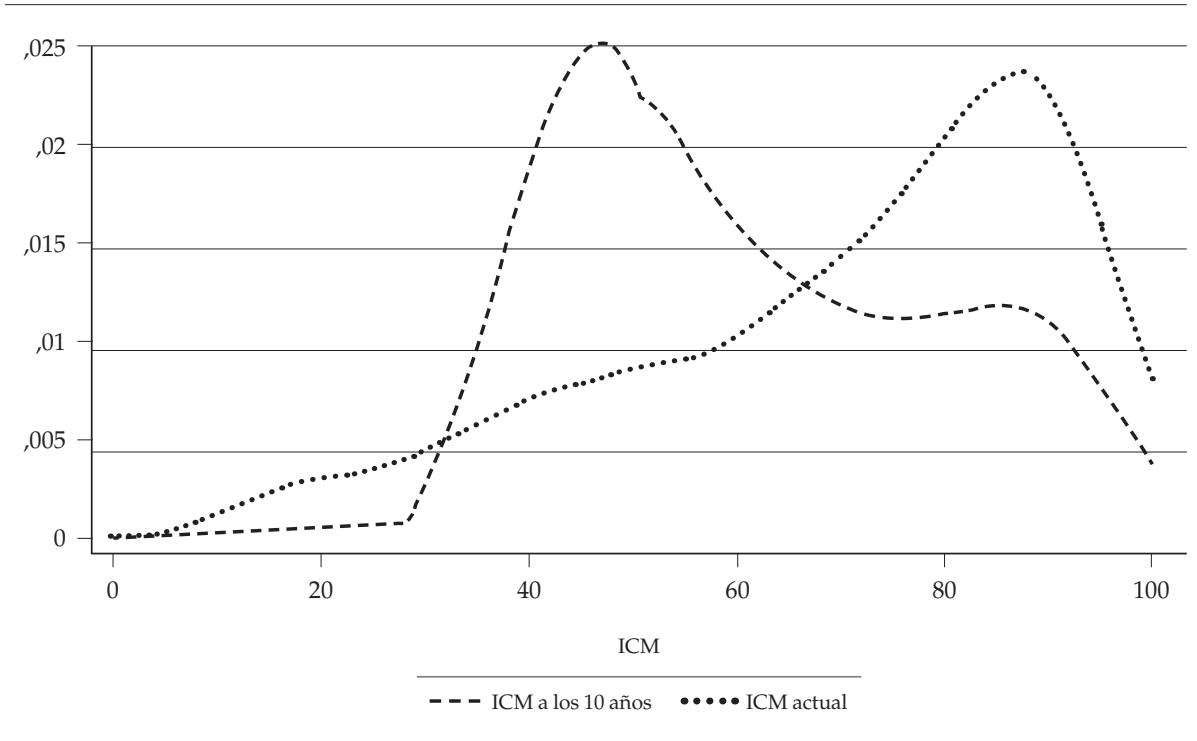

Figura 7. Distribución de los ICM en el pasado y en la actualidad Fuente: cálculos de los autores.

En la tabla 4 se muestra la matriz de transición en la calidad de vida. Se compara la situación actual del ICM con la que presentaba el hogar cuando el individuo tenía diez años. La tabla muestra que existe mayor movilidad en los niveles medios del índice de calidad de vida. Por otro lado, en el último quintil existe mayor persistencia: el $50 \%$ de los individuos cuyos padres estaban en este nivel de vida actualmente se mantienen en la misma posición.

Tabla 4. Matriz de movilidad socioeconómica, 2010

\begin{tabular}{lcccccc}
\hline & & \multicolumn{5}{c}{ Quintil del ICM del individuo en la actualidad } \\
\cline { 3 - 6 } & & 1 & 2 & 3 & 4 & 5 \\
\hline & 1 & 38,8 & 27,1 & 19,3 & 10,4 & 4,4 \\
Quintil & 2 & 32,5 & 26,1 & 19,1 & 14,0 & 8,3 \\
del ICM & 3 & 19,2 & 23,4 & 26,4 & 20,1 & 10,9 \\
en el hogar & & 6,4 & 15,7 & 22,4 & 29,4 & 26,1 \\
a los 10 años & 4 & 3,4 & 7,3 & 13,4 & 25,8 & 50,2 \\
& 5 & 3 & & & & \\
\hline
\end{tabular}

Fuente: cálculos de los autores. 
Los resultados de la tabla 4 dan una luz de optimismo, ya que en los quintiles más bajos existe mayor movilidad que en los más altos. No obstante, hay que anotar que en el segundo y tercer quintil la proporción de individuos que en la actualidad empeoraron es mayor con relación a los que mejoraron, o incluso a los que se mantuvieron en las mismas condiciones. Es decir, la mayor movilidad social en los niveles bajos del ICM es principalmente una movilidad descendente.

Complementario a lo anterior, si se comparan los cálculos presentados en la tabla 4 con la distribución de los ICM en la figura 7, se observa que las mayores densidades en el ICM actual se concentran en los puntos altos de la distribución, es decir, en los que presentan mayor riqueza material. De esta manera, los individuos con mayor prosperidad son los que experimentan en mayor grado una alta persistencia en las condiciones materiales.

Una pregunta que resulta del anterior hallazgo es la relación que existe entre la movilidad y la igualdad de oportunidades. El que los individuos que presentan mejor nivel de vida se mantengan más en su jerarquía, respecto de los que tienen menor nivel de vida, es un resultado que puede estar relacionado con la igualdad de oportunidades, en la medida en que existen ciertas condiciones que limitan la movilidad de las personas, como las características circunstanciales o las que son exógenas para el individuo. En la siguiente sección se evalúa dicha relación.

\subsection{Desigualdad de oportunidades}

En relación con la desigualdad de oportunidades, los cálculos se llevan a cabo al aplicar la formulación de Ferreira y Gignoux (2011) a la ecuación de la movilidad intergeneracional en la educación. Para relacionar la movilidad con la desigualdad de oportunidades se definieron tres grupos de individuos de acuerdo con su condición de movilidad en el ICM. Específicamente, los individuos que están sobre la diagonal son los que no tienen movilidad en el ICM, los que están por encima de la diagonal son los que tuvieron una mejoría o una movilidad ascendente y, por ende, los que están por debajo de la diagonal son los que desmejoraron en el ICM respecto de la situación que presentaban sus padres, es decir, una movilidad descendente. En este punto se avanza respecto de otros trabajos en el contexto colombiano, en el que solo se enfocan en la movilidad ascendente.

Los cálculos del índice de desigualdad relativa de oportunidades (IOP) se muestran en la tabla 5. Las estimaciones se hicieron tomando como base condiciones circunstanciales, como la educación de los padres y la condición étnica de los individuos. 
Tabla 5. Índice de desigualdad relativa de oportunidades, IOP, según sexo y área de residencia, 2010

\begin{tabular}{llcc}
\hline \multicolumn{1}{r}{ Tipo de movilidad } & Hombres & Mujeres \\
\hline \multirow{3}{*}{ IOP } & Movilidad descendente & 23,9 & 25,4 \\
& No movilidad & 36,6 & 44,2 \\
& Movilidad ascendente & 15,8 & 15,6 \\
\hline \multirow{2}{*}{ IOP } & Movilidad descendente & Rural & Urbano \\
& No movilidad & 22,6 & 20,3 \\
& Movilidad ascendente & 9,8 & 33,4 \\
\hline
\end{tabular}

Fuente: cálculos de los autores.

Lo que se debería esperar, desde el punto de vista de las condiciones favorables para que se recompense el esfuerzo de las personas por alcanzar un mejor nivel de vida, es que las características circunstanciales no expliquen significativamente las desigualdades existentes. Sin embargo, esa no es la situación en el caso colombiano.

Los resultados demuestran que la desigualdad de oportunidades condiciona las posibilidades de movilidad. Con ello, se concluye que existe una transmisión intergeneracional de las desigualdades. Este fenómeno tiene una mayor incidencia en las áreas urbanas para los grupos que tienen movilidad ascendente y para los que no experimentan movilidad.

Específicamente, los individuos que continúan en una situación de calidad de vida similar a la de sus padres son aquellos para los cuales las condiciones circunstanciales han sido más determinantes en sus logros, en términos de calidad de vida. Este resultado es consistente tanto para la comparación entre hombres y mujeres como para la urbano-rural.

Las condiciones circunstanciales son, a su vez, más importantes en la explicación de la movilidad descendente que la ascendente. Esto es, características ajenas a la voluntad del individuo están asociadas mayormente a las posibilidades de un descenso en el nivel de vida, que a una mejoría en este. La comparación entre hombres y mujeres no muestra diferenciales amplios, excepto en el grupo de los que no presentan movilidad, en donde para las mujeres un 44,2\% de la desigualdad está explicada por las condiciones circunstanciales al nacer, mientras que para los hombres es el 36,6\%.

La comparación por regiones en esta sección solo puede hacerse para las áreas urbanas frente a las rurales, dado que la ECVMS no tiene representatividad a nivel regional. Los cálculos de los índices de oportunidades muestran 
que para los grupos de no movilidad y de movilidad ascendente el porcentaje de la desigualdad explicada por las circunstancias al nacer es mayor en el área urbana: para quienes no presentan movilidad social las circunstancias al nacer explican 33,4\% de las desigualdades existentes. En contraste, en el área rural solo explican un $9,8 \%$.

La importancia de estos resultados radica en que las condiciones que no controlan los individuos no deberían marcar diferencias en las posibilidades de mejoría en la calidad de vida de las personas. De no ser así, existirán diferencias en las oportunidades solo por ser mujer o provenir de áreas rurales, cuando en realidad estas deberían responder a los esfuerzos propios. En otras palabras, el papel que cumple el esfuerzo propio del individuo en torno de las posibilidades de ascenso en las condiciones socioeconómicas estaría mediado por esas condiciones ajenas a la voluntad de las personas.

En relación con el caso de Estados Unidos, Chetty et al. (2013) documentan que la movilidad depende significativamente de la región de origen de los individuos. Específicamente, las posibilidades de presentar una movilidad ascendente en San Francisco, Salt Lake City, New York, Boston o Houston son mucho mayores que las de Memphis, Atlanta o Charlotte. En este sentido, los resultados del presente documento están en línea con lo presentado por Chetty et al. (2013). Específicamente, para los individuos que presentan movilidad ascendente el índice de desigualdad de oportunidades es más alto en el área urbana que en el área rural. También se podría argumentar que para quienes se ubican en las áreas más prósperas, las condiciones circunstanciales ayudan más a que se logre tener movilidad ascendente.

\section{Conclusiones}

Colombia presenta amplias desigualdades regionales, así como índices de persistencia de la pobreza que son bastante notables. El estudio de las desigualdades, sin embargo, no es suficiente para caracterizar las posibilidades de éxito o ascenso social de los individuos. En el presente trabajo se estudia la movilidad social, específicamente la movilidad intergeneracional de la educación, para caracterizar las regiones del país según las condiciones de movilidad ascendente o descendente de los individuos.

De acuerdo con los resultados, y en concordancia con otros estudios, se encuentra que el país presenta bajos índices de movilidad. Esos índices son heterogéneos en las regiones del país, encontrándose que Bogotá presenta los mejores resultados, mientras que la región Pacífica, los peores. Dicha relación 
es relativamente robusta en los diferentes ejercicios presentados, en el sentido en que a mayor prosperidad, mayor movilidad social.

De los cálculos de los índices de movilidad promedio y por cuantiles, se concluye que existe una significativa transmisión intergeneracional del logro educativo, ya que los años de educación alcanzados por los hijos están fuertemente asociados con los de los padres. Además, se encuentra que existe una transmisión intergeneracional del logro en las condiciones materiales, y que quienes experimentan movilidad en esta dimensión se caracterizan por presentar movilidad descendente, concentrándose en la parte baja de la distribución del ICM. Por su parte, quienes presentan mayor persistencia en el grado de riqueza están concentrados en la parte alta de la distribución. De esta manera, se confirma la máxima de que "es mejor ser rico que pobre".

Otra relación que se estudia es la de movilidad social con las condiciones de desigualdad, encontrándose que a mayor desigualdad, hay menos movilidad. Corak (2013) estudió esta relación en el contexto internacional refiriéndose a lo que se denomina la "Curva del Gran Gatsby". Siguiendo la argumentación de Corak (2013), las desigualdades en el ingreso y la movilidad social están mediadas por la desigualdad de oportunidades, que sería el grado en que la desigualdad es transmitida por medio de las generaciones.

Para estudiar más a fondo la relación entre desigualdades en movilidad y desigualdades en el ingreso y las oportunidades, en el presente estudio se analizaron las posibilidades de movilidad ascendente y descendente, así como los índices de igualdad de oportunidades calculados con base en Ferreira y Gignoux $(2008,2011)$. De acuerdo con dicho análisis, existen características circunstanciales que condicionan las posibilidades de movilidad de los individuos, entre estas se cuentan la educación de los padres y la etnia. Por género no se encuentran diferencias notables en el efecto de dichas condiciones circunstanciales sobre las posibilidades de movilidad de los individuos. Según el origen urbano contra el rural sí hay variaciones en el grado de explicación de los factores no controlados por los individuos en sus posibilidades de ascenso en la escala social. Es decir, existe una transmisión intergeneracional de las desigualdades, que empeoran el escenario de la baja movilidad social y la transmisión intergeneracional de las condiciones materiales.

Estos últimos resultados son importantes y merecen especial atención porque las condiciones circunstanciales, sobre la cuales no tiene control la persona, no deberían ser relevantes para condicionar el progreso del individuo. En respuesta a esta situación, la política social debería estar encaminada a garantizar, con criterios de equidad, que se den las condiciones para que recompense el esfuerzo de las personas en alcanzar un mejor nivel de vida, sin que estén de por medio las características circunstanciales. Infortunadamente, ese ideal no 
se cumple en el caso de Colombia, lo cual puede ir en detrimento de una mejor asignación del talento humano y de la reducción de las desigualdades.

\section{Referencias}

Angulo, R., Gaviria, A., \& Páez, G. N. (2012). Movilidad social en Colombia. Documentos CEDE, 43.

Barros, R., Ferreira, F., Vega, J., \& Chanduvi J. (2009). Measuring Inequality of Opportunities in Latin America and the Caribbean. Washington, D.C.: The World Bank.

Behrman, J. R., Gaviria, A., Székely, M., Birdsall, N., \& Galiani, S. (2001). Intergenerational Mobility in Latin America [with Comments]. Economía, Journal of the Latin American and Caribbean Economic Association, 2(1), pp. 1-44.

Bernal, R., \& Camacho, A. (2012). La política de primera infancia en el contexto de la equidad y movilidad social en Colombia. Documentos CEDE, 43.

Bonilla, L. (2010). Movilidad inter-generacional en educación en las ciudades y regiones de Colombia. Documentos de trabajo sobre economía regional, 130, CEER.

Bourguignon, F., Ferreira, F., \& Menéndez, M. (2007). Inequality of Opportunity in Brazil. Review of Income Wealth, 53(4), 585-618.

Cartagena, K. (2003). Educación y movilidad intergeneracional en Colombia, 1929-1996. Estudios Económicos de Desarrollo Internacional, 3(2), 27-66.

Chetty, R., Hendren, N., Kline, P., \& Saez, E. (2013). The Economic Impacts of Tax Expenditures. Evidence from Spatial Variation Across the U.S. Mimeo, Departamento de Economía.

Corak, M. (2013). Income Inequality, Equality of Opportunity, and Intergenerational Mobility. The Journal of Economic Perspectives, 27(3), 79-102.

Dahan, M., \& Gaviria, A. (1999). Sibling Correlations and Social Mobility in Latin America. IDB Working Paper, 395.

Eide, R., \& Showalter, M. (1999). Factors Affecting the Transmission of Earnings Across Generations: A Quantile Regression Approach. The Journal of Human Resources, 34(2), 253-267.

Espinoza, V. (2007). Movilidad social y trayectorias ocupacionales en Buenos Aires. Continuidades, rupturas y paradojas en los noventa. Mimeo.

Ferreira, F., \& Gignoux, J. (2008). The Measurement of Inequality of Opportunity: Theory and an application to Latin America. Policy Research Working Paper, 4659. The World Bank Development Research Group. 
Ferreira, F., \& Gignoux, J. (2011). The measurement of educational inequality: Achievement and Opportunity. Working Paper Series, 240. Society for the Study of Economic Inequality-ECINEQ.

Ferreira, F., \& Meléndez, M. (2012). Desigualdad de resultados y oportunidades en Colombia 1997-2010. Documentos CEDE, 40.

Flórez, C., Espinosa, F., \& Sánchez, L. (2008). Diseño del índice SISBEN en su tercera versión. Mimeo, Departamento Nacional de Planeación.

Galvis, L. A., \& Meisel, A. (2010). Persistencia de las desigualdades regionales en Colombia: Un análisis espacial. Documentos de trabajo sobre economía regional, 120, CEER.

Gaviria, A. (2002). Los que suben y los que bajan. Educación y movilidad social en Colombia. Bogotá: Ediciones Alfaomega-Fedesarrollo.

Gómez, M., Galvis, L., \& Aroca, P. (2013). Conmutación de larga distancia en Chile: el efecto de la calidad del empleo sobre la distancia y la probabilidad de conmutar. (Tesis de maestría, Universidad Católica de Chile, Santiago, Chile).

Koenker, R., \& Bassett, G. (1978). Regression Quantiles. Econometrica, 46(33), 33-50.

Lelli, S. (2001). Factor Analysis vs. Fuzzy Sets Theory: Assessing the influence of Different Techniques on Sen's Functioning Approach. Discussions Paper Series DPS, 01, 21.

Londoño, J. (2011). Movilidad social, preferencias redistributivas y felicidad en Colombia. Revista Desarrollo y Sociedad, 68, 171-212.

Nina, E., Grillo, S., \& Malaver, C. (2003). Movilidad social y transmisión de la pobreza en Bogotá. Economía y desarrollo, 2(2), 119-156.

Prais, S. J. (1955). Measuring Social Mobility. Journal of the Royal Statistical Society. Series A (General), 118(1), 56-66.

Roemer, J. E. (1998): Equality of Opportunity. Cambridge: Harvard University Press.

Sen, A. (1998). Capacidad y bienestar. En Nussbaum, M. \& Sen, A. (Comp.) La calidad de vida (pp. 95-100). México D.F.: Fondo de Cultura Económica.

Torche, F., \& Spilerman, S. (2009). Intergenerational Influences of Wealth in Mexico. Latin American Research Review, 44(3), pp. 75-101.

Viáfara, C., Estacio, A., \& González, L. (2010). Condición étnico-racial, género y movilidad social en Bogotá, Cali y el agregado de las trece áreas metropolitanas en Colombia: un análisis descriptivo y econométrico. Revista Sociedad y Economía, 18, 113-136. 


\section{Anexos}

\section{Anexo 1}

\section{Estadísticas descriptivas de la Encuesta de Calidad de Vida}

A. Año 2003

\begin{tabular}{lccc}
\hline \multicolumn{1}{c}{ Grupo étnico } & Frecuencia & Porcentaje & Acumulado \\
\hline Ninguno & 26922 & 91,96 & 91,96 \\
Indígena & 1834 & 6,26 & 98,22 \\
Afro otros & 521 & 1,78 & 100 \\
Total & 29277 & 100 & \\
\hline
\end{tabular}

\begin{tabular}{lccccc}
\hline \multicolumn{1}{c}{ Variables de la ECV } & Observaciones & Media & Desviación estándar & Mínimo & Máximo \\
\hline Edad & 29277 & 42,67 & 10,91 & 25 & 65 \\
Mujer & 29277 & 0,55 & 0,50 & 0 & 1 \\
Origen urbano & 29277 & 0,63 & 0,48 & 0 & 1 \\
Años educación padre & 26343 & 4,58 & 4,47 & 0 & 16 \\
Años educación madre & 28445 & 4,07 & 3,87 & 0 & 16 \\
Años educación hijo & 29277 & 8,38 & 5,12 & 0 & 20 \\
\hline
\end{tabular}

Fuente: cálculos propios con base en ECV, 2003

B. Año 2010

\begin{tabular}{lrcc}
\hline \multicolumn{1}{c}{ Grupo étnico } & Frecuencia & Porcentaje & Acumulado \\
\hline Ninguno & 13047 & 85,05 & 85,05 \\
Indígena & 1798 & 11,72 & 96,77 \\
Afro otros & 495 & 3,23 & 100 \\
Total & 15340 & 100 & \\
\hline
\end{tabular}

\begin{tabular}{lccccc}
\hline \multicolumn{1}{c}{ Variables de la ECV } & Observaciones & Media & Desviación estándar & Mínimo & Máximo \\
\hline Edad & 15340 & 43,18 & 10,98 & 25 & 65 \\
Mujer & 15340 & 0,54 & 0,50 & 0 & 1 \\
Origen urbano & 15340 & 0,54 & 0,50 & 0 & 1 \\
Años educación padre & 15340 & 2,97 & 3,22 & 0 & 16 \\
Años educación madre & 15340 & 3,02 & 3,16 & 0 & 16 \\
Años educación hijo & 15340 & 7,56 & 4,90 & 0 & 21 \\
\hline
\end{tabular}

Fuente: cálculos propios con base en ECV, 2010 


\section{Anexo 2}

\section{Estadísticas descriptivas de la Encuesta de Calidad de Vida y Movilidad Social, 2010}

A. Características del hogar cuando el individuo tenía 10 años de edad

\begin{tabular}{lccccc}
\hline Variables de la ECVMS & Observaciones & Media & Desviación estándar & Mínimo & Máximo \\
\hline Lavadora & 2726 & 0,05 & 0,21 & 0 & 1 \\
Nevera & 2726 & 0,26 & 0,44 & 0 & 1 \\
Licuadora & 2726 & 0,32 & 0,47 & 0 & 1 \\
Estufa & 2726 & 0,30 & 0,46 & 0 & 1 \\
Plancha & 2726 & 0,47 & 0,50 & 0 & 1 \\
Horno & 2726 & 0,08 & 0,27 & 0 & 1 \\
TV a color & 2726 & 0,10 & 0,30 & 0 & 1 \\
Equipo de sonido & 2726 & 0,14 & 0,34 & 0 & 1 \\
Aspiradora & 2726 & 0,04 & 0,19 & 0 & 1 \\
Aire acondicionado & 2726 & 0,01 & 0,10 & 0 & 1 \\
Ventilador & 2726 & 0,14 & 0,35 & 0 & 1 \\
Auto & 2726 & 0,07 & 0,26 & 0 & 1 \\
Casa de recreo & 2726 & 0,11 & 0,31 & 0 & 1 \\
Bicicleta & 2726 & 0,23 & 0,42 & 0 & 1 \\
\hline
\end{tabular}

Fuente: cálculos propios con base en ECVMS, 2010

\begin{tabular}{lccc}
\hline $\begin{array}{c}\text { Sitio de donde obtiene el agua para prepa- } \\
\text { rar los alimentos }\end{array}$ & Frecuencia & Porcentaje & Acumulado \\
\hline Acueducto público & 10 & 0,37 & 0,37 \\
Acueducto comunal o veredal & 15 & 0,56 & 0,93 \\
Pozo con bomba & 29 & 1,07 & 2,00 \\
Pozo sin bomba, jagüey & 896 & 33,19 & 35,19 \\
Agua lluvia & 67 & 2,48 & 37,67 \\
Río, quebrada, manantial o nacimiento & 300 & 11,11 & 48,78 \\
Pila pública & 81 & 3,00 & 51,78 \\
Carro tanque & 255 & 9,44 & 61,22 \\
Aguatero & 1047 & 38,78 & 100,00 \\
Total & 2700 & 100 & \\
\hline
\end{tabular}

Fuente: cálculos propios con base en ECVMS, 2010 


\begin{tabular}{lccc}
\hline \multicolumn{1}{c}{ Tipo de servicio sanitario } & Frecuencia & Porcentaje & Acumulado \\
\hline Inodoro conectado a alcantarillado & 776 & 28,83 & 28,83 \\
Inodoro conectado a pozo séptico & 11 & 0,41 & 29,23 \\
Inodoro sin conexión & 304 & 11,29 & 40,53 \\
Letrina & 152 & 5,65 & 46,17 \\
Bajamar & 561 & 20,84 & 67,01 \\
No tiene servicio sanitario & 888 & 32,99 & 100,00 \\
Total & 2692 & 100 & \\
\hline
\end{tabular}

Fuente: cálculos propios con base en ECVMS, 2010

\begin{tabular}{lrcc}
\hline \multicolumn{1}{c}{ Material predominante de los pisos } & Frecuencia & Porcentaje & Acumulado \\
\hline Alfombra & 1060 & 39,17 & 39,17 \\
Madera pulida y lacada, parqué & 841 & 31,08 & 70,25 \\
Mármol & 284 & 10,50 & 80,75 \\
Baldosa, vinilo, tableta, ladrillo & 398 & 14,71 & 95,45 \\
Madera burda, tabla, tablón, otro vegetal & 4 & 0,15 & 95,60 \\
Cemento, gravilla & 104 & 3,84 & 99,45 \\
Tierra, arena & 15 & 0,55 & 100,00 \\
Total & 2706 & 100 & \\
\hline
\end{tabular}

Fuente: cálculos propios con base en ECVMS, 2010

B. Características del hogar en la actualidad

\begin{tabular}{lccccc}
\hline Variables de la ECVMS & Observaciones & Media & Desviación estándar & Mínimo & Máximo \\
\hline Lavadora & 3128 & 0,44 & 0,50 & 0 & 1 \\
Nevera & 3128 & 0,75 & 0,43 & 0 & 1 \\
Licuadora & 3128 & 0,77 & 0,42 & 0 & 1 \\
Estufa & 3128 & 0,78 & 0,41 & 0 & 1 \\
Plancha & 3128 & 0,67 & 0,47 & 0 & 1 \\
Horno & 3128 & 0,22 & 0,41 & 0 & 1 \\
TV a color & 3128 & 0,91 & 0,29 & 0 & 1 \\
Equipo de sonido & 3128 & 0,50 & 0,50 & 0 & 1 \\
Aspiradora & 3128 & 0,07 & 0,25 & 0 & 1 \\
Aire acondicionado & 3128 & 0,04 & 0,19 & 0 & 1 \\
& & & & & Continúa
\end{tabular}




\begin{tabular}{lccccc}
\hline Variables de la ECVMS & Observaciones & Media & Desviación estándar & Mínimo & Máximo \\
\hline Ventilador & 3128 & 0,34 & 0,47 & 0 & 1 \\
Auto & 3128 & 0,13 & 0,34 & 0 & 1 \\
Casa de recreo & 3128 & 0,06 & 0,23 & 0 & 1 \\
Moto & 3128 & 0,17 & 0,38 & 0 & 1 \\
Horno microondas & 3128 & 0,16 & 0,36 & 0 & 1 \\
Calentador & 3128 & 0,17 & 0,38 & 0 & 1 \\
Computador & 3128 & 0,27 & 0,44 & 0 & 1 \\
Parabólica & 3128 & 0,41 & 0,49 & 0 & 1 \\
Internet & 3128 & 0,18 & 0,39 & 0 & 1 \\
\hline
\end{tabular}

Fuente: cálculos propios con base en ECVMS, 2010

\begin{tabular}{lccc}
\hline \multicolumn{1}{c}{$\begin{array}{c}\text { Sitio de donde obtiene el agua para } \\
\text { preparar los alimentos }\end{array}$} & Frecuencia & Porcentaje & Acumulado \\
\hline Acueducto público & 27 & 0,87 & 0,87 \\
Acueducto comunal o veredal & 23 & 0,74 & 1,60 \\
Pozo con bomba & 5 & 0,16 & 1,76 \\
Pozo sin bomba, jagüey & 215 & 6,89 & 8,66 \\
Agua lluvia & 104 & 3,33 & 11,99 \\
Río, quebrada, manantial o nacimiento & 82 & 2,63 & 14,62 \\
Pila pública & 59 & 1,89 & 16,51 \\
Carro tanque & 600 & 19,24 & 35,75 \\
Aguatero & 2004 & 64,25 & 100 \\
Total & 3119 & 100 & \\
\hline
\end{tabular}

Fuente: cálculos propios con base en ECVMS, 2010

\begin{tabular}{lccc}
\hline \multicolumn{1}{c}{ Tipo de servicio sanitario } & Frecuencia & Porcentaje & Acumulado \\
\hline Inodoro conectado a alcantarillado & 209 & 6,66 & 6,66 \\
Inodoro conectado a pozo séptico & 9 & 0,29 & 6,95 \\
Inodoro sin conexión & 39 & 1,24 & 8,19 \\
Letrina & 195 & 6,22 & 14,41 \\
Bajamar & 833 & 26,55 & 40,96 \\
No tiene servicio sanitario & 1852 & 59,04 & 100 \\
Total & 3137 & 100 & \\
\hline
\end{tabular}

Fuente: cálculos propios con base en ECVMS, 2010 


\begin{tabular}{lrcc}
\hline \multicolumn{1}{c}{ Material predominante de los pisos } & Frecuencia & Porcentaje & Acumulado \\
\hline Alfombra & 236 & 7,52 & 7,52 \\
Madera pulida y lacada, parqué & 1340 & 42,72 & 50,24 \\
Mármol & 94 & 3,00 & 53,24 \\
Baldosa, vinilo, tableta, ladrillo & 1310 & 41,76 & 95,00 \\
Madera burda, tabla, tablón, otro vegetal & 19 & 0,61 & 95,60 \\
Cemento, gravilla & 100 & 3,19 & 98,79 \\
Tierra, arena & 38 & 1,21 & 100 \\
Total & 3137 & 100 & \\
\hline
\end{tabular}

Fuente: cálculos propios con base en ECVMS, 2010 
